COMMUNICATIONS IN

ANALYSIS AND GEOMETRY

Volume 11, Number 3, 565-597, 2003

\title{
Stability, Energy Functionals, and Kähler-Einstein Metrics
}

\author{
D. H. Phong And Jacob Sturm
}

\section{Introduction.}

Starting with the work of Yau [Y1], Donaldson [D1], and Uhlenbeck-Yau [UY], the notion of stability has revealed itself under many guises to be closely related to the existence of canonical metrics in Kähler geometry. The equivalence between Hermitian-Einstein metrics on vector bundles and Mumford stability was proved by Donaldson and Uhlenbeck-Yau in [D1] and [UY], while the existence of Kähler-Einstein metrics was conjectured in the early 1980's by Yau [Y2] to be equivalent to stability in geometric invariant theory. At the present time, the Yau conjecture has been at least partially confirmed. The existence of Kähler-Einstein metrics has been shown to imply $K$-stability and $C M$-stability by Tian [T2], and more recently to imply Chow-Mumford stability by Donaldson [D2].

In moduli theory, a non-zero vector $\operatorname{Chow}(A)$ in a vector space $\mathbf{C}^{N+1}$ is associated to geometric objects $A$ (such as vector bundles or varieties). The vector $\operatorname{Chow}(A)$ is defined up to multiplicative constants, and its $G L(N+1)$ orbit inside $\mathbf{P}^{N}$ uniquely determines $A$. Thus the moduli space can be constructed as the space of orbits. The vector $\operatorname{Chow}(A)$ is said to be stable if $S L(N+1) \cdot \operatorname{Chow}(A) \subseteq \mathbf{C}^{\mathbf{N}+\mathbf{1}}$ is closed and the stabilizer of $\operatorname{Chow}(A)$ is finite. Stability is of particular importance, since Geometric Invariant Theory guarantees that the space of stable orbits has the structure of an algebraic variety $[\mathrm{Mu}]$. On the other hand, the emergence of stability as a necessary condition for the existence of canonical metrics can be quite subtle. Basic to Tian's approach is a remarkable asymptotic equivalence between a Lagrangian for constant scalar curvature metrics, namely the Mabuchi energy, and a norm $\|\cdot\|_{Q}$ on the space of Chow vectors which he constructed by $\partial \bar{\partial}$ methods and identified with suitable Quillen metrics [T1,

\footnotetext{
${ }^{1}$ Supported in part by the National Science Foundation under grants DMS-9800783 and DMS-01-00410.
} 
T2]. Quillen metrics had been related earlier to Lagrangians for HermitianEinstein metrics by Donaldson [D3]. The asymptotic equivalence between $\|\cdot\|_{Q}$ and the Mabuchi energy becomes exact for hypersurfaces in $\mathbf{P}^{n+1}$. Donaldson [D2] relies instead on the concept of balanced manifolds and Lu's recent evaluation [Lu1] of the Tian-Yau-Zelditch expansion for the Bergman kernel on positive line bundles.

In this paper, building on the earlier work of Yau [Y3, Y4], Tian [T1,T2] and Zhang $[\mathrm{Z}]$, we construct a semi-norm $\|\cdot\|_{\#}$ which is defined on the full space $H^{0}(G r, O(d))$ of polynomials of degree $d$ on the Grassmann variety $G r$, and which gives exactly the Mabuchi functional when restricted to the space of Chow vectors, up to a specific current supported on the singular locus of the Chow variety. This new semi-norm can be described quite explicitly: Let $G r=G r\left(N-n-1, \mathbf{P}^{N}\right)$ be the space of $N-n-1$ projective planes in $\mathbf{P}^{N}, P \ell: G r\left(N-n-1, \mathbf{P}^{N}\right) \rightarrow \mathbf{P}\left(\wedge^{N-n} \mathbf{C}^{N+1}\right)$ be the Plücker imbedding, and $O(1)=P \ell^{*} H$, where $H$ is the hyperplane bundle on $\mathbf{P}\left(\wedge^{N-n} \mathbf{C}^{N+1}\right)$. Let $\omega_{G r}=P \ell^{*} \omega_{F S}$, where $\omega_{F S}$ is the Fubini-Study Kähler form on the space $\mathbf{P}\left(\Lambda^{N-n} \mathbf{C}^{N+1}\right)$. Let $d$ be an arbitrary positive integer, and denote by $m+1=(N-n)(n+1)$ and $D=\int_{G r} \omega_{G r}^{m+1}$ respectively the dimension and the volume of $G r$. Then for any $f \in H^{0}(G r, O(d))$, we define the norm $\|f\|_{\#}$ of $f$ by

$$
\begin{aligned}
\log \|f\|_{\#}^{2}= & \frac{(m+1)}{(m+2)(d-1)} \cdot \frac{1}{D} \int_{Z} \log \left(\frac{\omega_{G r}^{m} \wedge \partial \bar{\partial} \frac{|f(z)|^{2}}{|P \ell(z)|^{2 d}}}{\omega_{G r}^{m+1}}\right) \omega_{G r}^{m} \\
& +\frac{d-m-2}{(m+2)(d-1)} \cdot \frac{1}{D} \int_{G r} \log \frac{|f(z)|^{2}}{|P \ell(z)|^{2 d}} \omega_{G r}^{m+1}
\end{aligned}
$$

where $Z=\{z \in G r: f(z)=0\}$.

One easily sees that $\|\cdot\|_{\#}$ defines a semi-norm on the finite dimensional vector space $H^{0}(G r, O(d))$ : That is, $\|\lambda \cdot f\|_{\#}=|\lambda| \cdot\|f\|_{\#}$ for every $\lambda \in \mathbf{C}$ and $f \in H^{0}(G r, O(d))$. Moreover, $\|f\|_{\#} \geq 0$ for all $f$. On the other hand, $\|\cdot\|_{\#}$ is not a norm since for $d>1$ there exist non-zero elements $f \in H^{0}(G r, O(d))$ such that that $\|f\|_{\#}=0$.

Our main result can be described as follows. Let $X \subseteq \mathbf{P}^{N}$ be a smooth algebraic variety of dimension $n, Z \subseteq G r$ be the corresponding Chow variety, and let $\operatorname{Chow}(X)=f \in H^{0}(G r, O(d))$ be a defining section for $Z$. Note that $Z$ is a singular variety. We assume that the embedding $X \subseteq \mathbf{P}^{N}$ is generic in the sense defined in $\S 6$. 
Let $Z_{s}$ be the singular locus of $Z$, and let $Y_{s}=\left\{(x, z) \in X \times Z_{s} ; x \in z\right\} \subset$ $\mathbf{P}^{N} \times G r$. Let $\omega_{Z}$ be the restriction of $\omega_{G r}$ to $Z_{0}=Z \backslash Z_{s}$. Then the Ricci curvature $\operatorname{Ric}\left(\omega_{Z}\right)$ of $\omega_{Z}$ is a smooth $(1,1)$ form on $Z_{0}$. Let $s\left(\omega_{Z}\right)$ be the scalar curvature of $\omega_{Z}, V=\operatorname{vol}(Z)$, and define

$$
\mu(Z)=\frac{1}{V} \int_{Z_{0}} s\left(\omega_{Z}\right) \omega_{Z}^{m}
$$

Let $\left[Y_{s}\right]$ be the current corresponding to $Y_{s}$ (see $\S 6$ for the precise definition) and define

$$
\operatorname{deg}\left(Y_{s}\right)=\frac{1}{V}\left\langle\left[Y_{s}\right], \omega_{Z}^{m-1}\right\rangle
$$

Let $\omega$ be the restriction of the Fubini-Study metric to $X$, and let $\nu_{\omega}(\varphi)$ be the Mabuchi energy on $X$ (see $\S 5$ for the precise definition). For each $\sigma \in G L(N+1)$, let $\varphi_{\sigma}$ and $\Phi_{\sigma}$ be the following functions on $\mathbf{P}^{N}$ and $G r$ respectively

$$
\varphi_{\sigma}(z)=\log \frac{|\sigma z|^{2}}{|z|^{2}}, \quad \Phi_{\sigma}(z)=\log \frac{|P \ell(\sigma z)|^{2}}{|P \ell(z)|^{2}}
$$

Define a generalized Mabuchi energy $\nu_{\omega}^{\#}\left(\varphi_{\sigma}\right)$ by

$$
\begin{aligned}
\nu_{\omega}^{\#}\left(\varphi_{\sigma}\right)= & \nu_{\omega}\left(\varphi_{\sigma}\right)+\frac{1}{V}\left\langle\left[Y_{s}\right], \Phi_{\sigma} \sum_{i=0}^{m-1} \omega_{Z}^{i} \sigma^{*} \omega_{Z}^{m-1-i}\right\rangle \\
& -\frac{D}{V} \cdot \frac{m \operatorname{deg}\left(Y_{s}\right)}{m+1} \cdot \log \frac{\|\sigma \cdot \operatorname{Chow}(X)\|^{2}}{\|\operatorname{Chow}(X)\|^{2}}
\end{aligned}
$$

where $\|\cdot\|$ is the norm defined in (4.1) below. Then

Theorem 1. For $\sigma \in S L(N+1, \mathbf{C})$ we have

$$
\nu_{\omega}^{\#}\left(\varphi_{\sigma}\right)=\frac{D(m+2)(d-1)}{V(m+1)} \log \frac{\|\sigma \cdot \operatorname{Chow}(X)\|_{\#}^{2}}{\|\operatorname{Chow}(X)\|_{\#}^{2}}
$$

An interesting new notion emerges from the proof of Theorem 1, namely the generalized Mabuchi energy $\nu_{\omega_{Z}}^{\#}\left(\Phi_{\sigma}\right)$ of the singular Chow variety $Z$ (see (6.3) for its precise definition). The Chow variety $Z$ contains a singular locus $Z_{s}$ of codimension 1 , and the generalized Mabuchi energy $\nu_{\omega_{Z}}^{\#}\left(\Phi_{\sigma}\right)$ is defined accordingly as consisting of the usual Mabuchi energy $\nu_{\omega_{Z}}\left(\Phi_{\sigma}\right)$ of the regular part $Z_{0}=Z \backslash Z_{s}$, together with additional current terms due to $Z_{s}$ (or more precisely, $\left[Y_{s}\right]$ ). Theorem 1 is then the consequence of two 
results, which may be interesting in their own right (c.f. Lemmas 6.1 and 6.2). The first result is that the right hand side of (1.3) can be equated with the generalized Mabuchi energy $\nu_{\omega_{Z}}^{\#}\left(\Phi_{\sigma}\right)$ of the singular Chow variety $Z$. The second result is that the Mabuchi energy $\nu_{\omega}\left(\varphi_{\sigma}\right)$ of the projective variety $X$ can be equated with the Mabuchi energy $\nu_{\omega_{Z}}\left(\Phi_{\sigma}\right)$ of the regular part $Z_{0}$ of its Chow Variety $Z$.

As mentioned above, a similar formula to (1.3) with an asymptotic bounded error has been proved by Tian [T1], with $\|\cdot\|_{\#}$ replaced by the Quillen metric $\|\cdot\|_{Q}$ of a certain virtual line bundle. In the case where $X \subseteq \mathbf{P}^{N}$ is a smooth hypersurface, the version of (1.3) with $\|\cdot\|_{Q}$ also becomes exact, and it is likely that the two notions $\|\cdot\|_{\#}$ and $\|\cdot\|_{Q}$ coincide. This issue is more complicated for higher codimensions, not just because the error term in the version of (1.3) with $\|\cdot\|_{Q}$, but also because $\|\cdot\|_{Q}$ is presently defined only on the space of Chow vectors, and not yet on the whole of $H^{0}(G r, O(d))$.

Besides the fact that it is completely explicit and satisfies the relation (1.3) exactly, the semi-norm $\|f\|_{\#}$ in (1.1) has several attractive features which may be valuable in future investigations of the relation between various notions of stability and the existence of Kähler-Einstein metrics. Indeed, the existence of Kähler-Einstein metrics is known to imply the boundedness from below of energy functionals (see Siu-Yau [SY], Bando-Mabuchi $[\mathrm{BM}]$, and Ding-Tian [DT]). Theorem 1 suggests that the boundedness from below of the Mabuchi energy functional can eventually be related to the boundedness from below of the norm $\|f\|_{\#}$, which is defined even for singular varieties. Furthermore, $\|f\|_{\#}$ is degenerate, so that the condition that $\|\sigma \cdot \operatorname{Chow}(X)\|_{\#} /\|\operatorname{Chow}(X)\|_{\#} \rightarrow \infty$ appears to be a stronger notion of stability than the usual notion of Chow-Mumford stability. It may be closely related to the notion of $C M$-stability introduced earlier by Tian [T1].

Our approach is based on an exact evaluation of the derivative of $\log \| \sigma(t)$. Chow $(X) \|$ along each 1 - parameter orbit of $S L(N+1)$. This method appears to be technically simpler than the approach in [T1][T2], which is based instead on the evaluation of $\partial \bar{\partial} \log \|\sigma \cdot \operatorname{Chow}(X)\|$ on $S L(N+1)$.

Our method applies equally well to other contexts, namely to the component $L(h, k)$ of the Donaldson functional $\mathbf{L}(k, h)$ for the existence of HermitianEinstein metrics on vector bundles, and to the component $F_{\omega}^{0}(\varphi)$ of the Lagrangian $F_{\omega}(\varphi)$ for Kähler-Einstein metrics. In the case of $F_{\omega}^{0}(\varphi)$, an exact relation of the form (1.3) had been obtained by Zhang [Z], using the theory of Deligne pairings [D]. Asymptotic relations modulo $O(1)$ terms had 
been obtained by Paul $[\mathrm{P}]$ and Wang [W] respectively for $F_{\omega}^{0}(\varphi)$ and for $L(h, k)$. We shall use our approach to give a unified and simpler proof of these earlier results. We present these results in $(\S 2-\S 4)$ before proceeding to the more complicated calculation of the Mabuchi energy, and take the opportunity to mildly strengthen earlier results of Zhang [Z], Luo [Luo], and Wang $[\mathrm{W}]$ whenever it is readily possible to do so by our methods. In particular, we eliminate the $O(1)$ error terms in [W] by showing that $L(h, k)$, restricted to $G=S L(N, \mathbf{C})$, equals exactly the log of the Gieseker point in a suitable norm. Wang [W] had shown that a vector bundle can be uniquely balanced if and only if its Gieseker point is stable. Similarly, we show that a manifold can be uniquely balanced if and only if its Chow point is stable.

\section{The Donaldson energy functional and balanced bundles.}

Let $(X, \omega)$ be a Kähler manifold and $\pi: E \rightarrow X$ a vector bundle of rank $r$. The Donaldson functional $\mathbf{L}(h, k)$ is defined as follows. Let $h, k$ be two hermitian metrics on $E$. Since the space of hermitian metrics is convex, we can connect $k$ to $h$ be a smooth path of hermitian metrics $h_{t}, 0 \leq t \leq 1$, $h_{0}=h, h_{1}=k$. Let $R_{t}$ be the curvature of $h_{t}$. Then the Donaldson functional $\mathbf{L}(h, k)$ is defined by

$$
\mathbf{L}(h, k)=\int_{0}^{1} d t \int_{X} i \operatorname{tr}\left(h_{t}^{-1} \partial_{t} h_{t} \cdot R_{t}\right) \frac{\omega^{n-1}}{(n-1) !}-\frac{c}{V} \int_{X} \log \left(\operatorname{det}\left(k^{-1} h\right)\right) \frac{\omega^{n}}{n !}
$$

where the constant $c$ is given by $c=\frac{2 \pi n}{r} \int_{X} c_{1}(E) \wedge \omega^{n-1}$. In this section, $V=\operatorname{vol}(X)=\int_{X} \omega^{n}$ denotes the volume of $X$ with respect to the Kähler form $\omega$.

Assume $E^{*}$ is generated by sections $s_{1}, \ldots, s_{N} \in H^{0}\left(X, E^{*}\right)$. If $e \in E$ then $s(e)=\left(s_{1}(e), \ldots, s_{N}(e)\right) \in \mathbf{C}^{N}$ so $s \times \pi: E \hookrightarrow \mathbf{C}^{N} \times X$. Let $h$ be the metric on $E$ defined by $h(e)=\sum\left|s_{i}(e)\right|^{2}=|s(e)|^{2}$, where $|\cdot|$ is the usual norm on $\mathbf{C}^{N}$.

Fix $\left\{\tau_{1}, \ldots, \tau_{k}\right\}$, a basis for $H^{0}\left(X, \operatorname{det}\left(E^{*}\right)\right)$. Let $T$ be the matrix $T=$ $\left(a_{i_{1} \cdots i_{r}}^{\mu}\right) \in M$, defined by the equation

$$
s_{i_{1}} \wedge \cdots \wedge s_{i_{r}}=s_{i_{1} \cdots i_{r}}=\sum_{\mu} a_{i_{1} \cdots i_{r}}^{\mu} \tau_{\mu}
$$

where $M$ is the space of matrices $\left(A_{i_{1} \cdots i_{r}}^{\mu}\right)$ with $1 \leq i_{1}<\cdots i_{r} \leq N$ and $1 \leq \mu \leq k$. The Gieseker point of $s=\left(s_{1}, \ldots, s_{N}\right)$ is the point $[T]=$ $\left(a_{i_{1} \cdots i_{r}}^{\mu}\right) \in \mathbf{P}(M)$. Then $[T]$ uniquely determines the image of $E$ in $\mathbf{C}^{N} \times X$. 
For $\sigma \in G$ we shall write $s^{\sigma}=\left(s_{1}, \ldots, s_{N}\right) \sigma$, and $h^{\sigma}(e)=\left|s^{\sigma}(e)\right|$. We define

$$
L(\sigma)=L\left(h, h_{\sigma}\right)=\frac{c}{V} \int_{X} \log \left(\frac{\operatorname{det}\left(h^{\sigma}\right)}{\operatorname{det}(h)}\right) \frac{\omega^{n}}{n !}
$$

which is the second term in the definition of the Donaldson functional $\mathbf{L}(k, h)$. In local coordinates, if $e_{1}, \ldots, e_{r}$ is a basis of smooth sections of $E \subseteq$ $\mathbf{C}^{N} \times U$, then for each $m$ such that $1 \leq m \leq r$, and for each $x \in U, e_{m}(x)=$ $e_{i m}(x)$ is a column vector in $\mathbf{C}^{N}$. Hence $A(x)=\left(e_{i m}(x)\right)$ is an $N \times r$ matrix, and $h$ is the $r \times r$ matrix $h={ }^{t} \bar{A} A$. Thus $\operatorname{det}(h)=\sum_{i_{1}<\cdots<i_{r}}\left|\operatorname{det}\left(A_{i_{1} \cdots i_{r}}\right)\right|^{2}$. Since $s_{i}(x)=\sum e_{i m}(x) e_{m}^{*}$ we get $\operatorname{det}(h)=\sum_{i_{1}<\cdots<i_{r}}\left|s_{i_{1} \cdots i_{r}}\right|^{2}$.

Thus

$$
\begin{aligned}
L(\sigma) & =\frac{c}{V} \int_{X} \log \left(\frac{\operatorname{det}\left(A(x)^{*} \sigma^{*} \sigma A(x)\right)}{\operatorname{det}\left(A(x)^{*} A(x)\right)}\right) \frac{\omega^{n}}{n !} \\
& =\frac{c}{V} \int_{X} \log \left(\frac{\sum\left|\left(a_{i_{1} \cdots i_{r}}^{\mu}\right)^{\sigma} \tau_{\mu}(x)\right|^{2}}{\sum\left|a_{i_{1} \cdots i_{r}}^{\mu} \tau_{\mu}(x)\right|^{2}}\right) \frac{\omega^{n}}{n !}
\end{aligned}
$$

where $A^{*}$ is the conjugate transpose of $A,\left(a_{i_{1} \cdots i_{r}}^{\mu}\right)^{\sigma}$ is the natural action of $G$ on $M$, and the summation is over all $\mu$ with $1 \leq \mu \leq k$ and all $1 \leq i_{1}<\cdots<i_{r} \leq N$. We note that the integral in (2.1) is finite. This suggests defining the following norm on the vector space $M$ : Set for each $a=\left(a_{i_{1} \cdots i_{r}}^{\mu}\right) \in M$

$$
\log \|a\|^{2}=\frac{1}{V} \int_{X} \log \sum\left|a_{i_{1} \cdots i_{r}}^{\mu} \tau_{\mu}(x)\right|^{2} \frac{\omega^{n}}{n !}
$$

It is easy to see that $\|\cdot\|$ is a continuous norm, and hence bounded on any compact subset of $M$. In terms of $\|\cdot\|$, the formula (2.1) can be restated as

Theorem 2. Let $E \subseteq \mathbf{C}^{N} \times X$ be a vector bundle of rank $r$, let $T$ be the Gieseker point of $E$, and let $h$ be the metric on $E$ defined by $h(e)=|e|_{\mathbf{C}^{N}}$. For $\sigma \in G=S L(N, \mathbf{C})$, let $h_{\sigma}$ be the metric on $E$ defined by $h_{\sigma}(e)=$ $|\sigma(e)|_{\mathbf{C}^{N}}$. Then

$$
L\left(h, h_{\sigma}\right)=c \log \frac{\|\sigma T\|^{2}}{\|T\|^{2}}
$$

Remark. This is slightly more precise than a result of Wang [W], who shows that for any norm $\|\cdot\|$ on $M$, one has $L\left(h, h_{\sigma}\right) \geq c \log \left(\|\sigma T\|^{2}\right)+C$ for some constant $C$. 
According to the theorem of Kempf-Ness $[\mathrm{KN}], E$ is Gieseker stable if and only if $\|\sigma T\|^{2}$ is a proper map from $G$ to $\mathbf{R}$ (the inverse image of a compact set is compact). This is equivalent to requiring that $\log \|\sigma T\|^{2}$ is bounded below by a positive constant and that $\lim _{\sigma \rightarrow \infty} \log \|\sigma T\|^{2}=\infty$ (ie, for every $B>0$ there exists a compact subset $K \subseteq G$ such that $L(\sigma) \geq B$ if $\sigma \notin K)$. Combining this with Theorem 2 we have the following corollary:

Corollary 2.1 Let $E \subseteq \mathbf{C}^{N} \times X$ be a vector bundle of rank $r$. Then $E$ is Gieseker stable if and only if the following conditions hold:

1. $L(\sigma) \geq \epsilon>0$ for some $\epsilon$.

2. $\lim _{\sigma \rightarrow \infty} L(\sigma)=\infty$.

Definition 2.1 We say $E \subseteq \mathbf{C}^{N} \times X$ is balanced if

$$
\frac{1}{V} \int_{X} A(x) A(x)^{*} d V=\frac{r}{N} \cdot I_{N \times N}
$$

where $A(x)=\left(a_{1}, \cdots a_{r}\right)$ is an orthonormal basis of $E_{x} \subseteq \mathbf{C}^{N}$. We say $E$ can be (uniquely) balanced if and only if there exists a (unique) $\sigma_{0} \in S U(N) \backslash G$ such that $\sigma_{0}(E)$ is balanced.

Example. Let $X=G r(r, N)$, the Grassmannian variety of all $r$ planes in $\mathbf{C}^{N}$. Let $E$ be the canonical vector bundle on $X$ of rank $r$. Then one easily sees that $X$ is balanced.

Theorem 3. (Wang) Let $E \subseteq \mathbf{C}^{N} \times X$ be a vector bundle. Then the bundle $E$ can be uniquely balanced if and only if its Gieseker point is stable.

Theorem 3 as well as Lemmas 2.1 and 2.2 below are due to Wang [W], under a slightly different formulation. He uses arguments from the theory of moment maps. Here we shall provide a direct calculation, along the lines followed later for the proof of Theorem 1.

Lemma 2.1 L has a critical point at $\sigma_{0} \in G$ if and only if $\sigma_{0}(E)$ is balanced.

Proof. Write $\sigma(t)=\exp (t c) \sigma_{0}$ where $\sigma_{0} \in G$ is fixed, and $c$ is traceless. Then

$$
\frac{d}{d t} \log \operatorname{det}\left(A^{*} \sigma^{*} \sigma A\right)=\operatorname{tr}\left(\left(A^{*} \sigma^{*} \sigma A\right)^{-1}\left(A^{*} \sigma^{*}\left(c^{*}+c\right) \sigma A\right)\right.
$$

Now $\sigma_{0}$ is a critical point if and only if $\frac{d}{d t} L\left(\exp (c t) \sigma_{0}\right)=0$ for all traceless $c$. Replacing $A$ by $A \sigma_{0}$ we may assume that $\sigma_{0}=I$. We may also choose our local sections $e_{1}, \ldots, e_{r}$ to be orthonormal. Then $A^{*} A=I$ and (2.2) implies 
that $\sigma_{0}$ is critical if and only if

$$
\int_{X} \operatorname{tr}\left(A^{*} u A\right) d V=0
$$

for all traceless hermitian $u$. This condition is equivalent to $E$ being balanced. Q.E.D.

Next we show $L(\sigma)$ is convex in the following sense: View $L(\sigma)$ as a function on the symmetric space $S U(N) \backslash G$. Then $S U(N) \backslash G$ is a Riemannian manifold whose geodesics are of the form $\exp (t c) \sigma_{0}$ where $c$ is a traceless matrix with the property $c^{*}=c$.

Lemma 2.2 Let $\sigma_{0} \in G$ and let $c$ be an arbitrary traceless self-adjoint matrix. Then

$$
\frac{d^{2}}{d t^{2}} L\left(\exp (t c) \sigma_{0}\right) \geq 0
$$

Proof. Differentiating (2.2) again gives

$$
\begin{gathered}
\frac{d^{2}}{d t^{2}} \log \operatorname{det}\left(A^{*} \sigma^{*} \sigma A\right)=\operatorname{tr}\left(\left(A^{*} \sigma^{*} \sigma A\right)^{-1}\left(A^{*} \sigma^{*}\left[c^{*}\left(c^{*}+c\right)+\left(c^{*}+c\right) c\right] \sigma A\right)\right) \\
-\operatorname{tr}\left(\left(A^{*} \sigma^{*} \sigma A\right)^{-1}\left(A^{*} \sigma^{*}\left(c^{*}+c\right) \sigma A\right)\left(A^{*} \sigma^{*} \sigma A\right)^{-1}\left(A^{*} \sigma^{*}\left(c^{*}+c\right) \sigma A\right)\right)
\end{gathered}
$$

We may assume that $\sigma_{0}=I$ and $A^{*} A=I$. Let $u=c+c^{*}=2 c$. Thus $u=u^{*}$ and $u$ is traceless. The preceding equation becomes

$$
\begin{gathered}
\frac{d^{2}}{d t^{2}} \log \operatorname{det}\left(A^{*} \sigma^{*} \sigma A\right)=\operatorname{tr}\left(A^{*}\left[u^{2}\right] A\right)-\operatorname{tr}\left(\left(A^{*}(u) A\right)\left(A^{*}(u) A\right)\right)= \\
\operatorname{tr}\left(A^{*} u\left(1-A A^{*}\right) u A\right)=\operatorname{tr}\left(\left(1-A A^{*}\right) u A A^{*} u^{*}\right)
\end{gathered}
$$

This last trace is non-negative since $u A A^{*} u^{*} \geq 0$ and $1-A A^{*} \geq 0$. To see this last inequality, let $\lambda$ be an eigenvalue of $A A^{*}$. Then $A A^{*} v=\lambda v$ for some non-zero vector $v$. Applying $A^{*}$ to both sides, and using the fact that $A^{*} A=I$, we get $A^{*} v=\lambda A^{*} v$. Thus $\lambda=1$ or $\lambda=0$. Hence all the eigenvalues of $1-A A^{*}$ are $\geq 0$ and $1-A A^{*} \geq 0$.

Proof of Theorem 3. Assume first that $L$ has a critical point $\sigma_{0}$. If $\sigma \in G$ is any other point, then we can join $\sigma_{0}$ to $\sigma$ by a geodesic. Lemma 2.2 implies that $L$, restricted to the geodesic, has a minimum at $\sigma_{0}$. Thus $L(\sigma) \geq L\left(\sigma_{0}\right)$, so $L$ is bounded below. Theorem 2 implies that $\|\sigma T\|$ is bounded below, and thus by definition the Gieseker point is semi-stable. If the critical point $\sigma_{0}$ is 
unique, then $L(\sigma)$ achieves its minimum at $\sigma_{0}$ and the same argument shows that $L$, restricted to any geodesic through $\sigma_{0}$, goes to infinity. Thus the Gieseker point is stable (by virtue of the one parameter subgroup criterion)

As for the converse, assume that $E$ is stable. Then the Kempf-Ness theorem says that $\log \|\sigma T\|$ is proper on $H$ and bounded below. Thus it has a critical point. The critical point is unique, for otherwise, $\log \|\sigma T\|$ would be constant on the geodesic joining two critical points and thus it could not be proper.

\section{The $F_{\omega}^{0}$ functional and balanced manifolds.}

Consider now a smooth projective variety $X \subset \mathbf{P}^{N}$ of degree $d$ and dimension $n$. Let $Z$ be the set of $(N-n-1)$-dimensional planes in $\mathbf{P}^{N}$ which intersect $X$. Then $Z$ is contained in $G r=G r\left(N-n-1, \mathbf{P}^{N}\right)$ and has codimension 1. Thus there exists a holomorphic section $f \in H^{0}(G r, O(d))$, unique up to scalar multiplication, which vanishes precisely on $Z$. The section $f$ defines then a point in $\mathbf{P} H^{0}(G r, O(d))$, which is called the Chow point of $X \hookrightarrow \mathbf{P}^{N}$, and usually denoted by $\operatorname{Chow}(X)$.

We shall apply the same method as in the previous section to prove the following:

Theorem 4. Let $X \subseteq \mathbf{C} P^{N}$ be a smooth projective variety. Then the Chow point of $X$ is stable if and only if there is a unique $\sigma_{0}$ in $S U(N+1) \backslash S L(N+$ $1, \mathbf{C})$ such that $\sigma_{0}(X)$ is balanced, i.e.,

$$
\frac{1}{\operatorname{vol}(X)} \int_{\sigma_{0}(X)}\left(\frac{\bar{z}_{j} z_{i}}{\left|z_{0}\right|^{2}+\cdots\left|z_{N}\right|^{2}}\right) \omega_{F S}^{n}=\frac{1}{N+1} \cdot \delta_{i j}
$$

Remark. This theorem is a mild strengthening of a result of Zhang [Z], who shows that balanced implies semi-stable and stable implies uniquely balanced. It also mildly strenghthens a similar theorem of Luo [Luo].

To prove Theorem 4 , we make use of the component $F_{\omega}^{0}(\varphi)$ of a Lagrangian $F_{\omega}(\varphi)$ for Kähler-Einstein metrics

$$
F_{\omega}(\varphi)=F_{\omega}^{0}(\varphi)-\log \left(\frac{1}{V} \int_{X} e^{h_{\omega}-\varphi} \omega^{n}\right)
$$

Here $\omega$ is a Kähler metric on $X, \varphi$ is a smooth function in the Kähler cone of $X, \omega_{\varphi}=\omega+\frac{\sqrt{-1}}{2 \pi} \partial \bar{\partial} \varphi, V=\operatorname{vol}(X)=\int_{X} \omega^{n}$, and $h_{\omega}$ is defined by $\operatorname{Ric}(\omega)-\omega=\frac{\sqrt{-1}}{2 \pi} \partial \bar{\partial} h_{\omega}, \int_{X} e^{h_{\omega}} \omega^{n}=\int_{X} \omega^{n}$. The component $F_{\omega}^{0}(\varphi)$ is 
defined by

$$
F_{\omega}^{0}(\varphi)=J_{\omega}(\varphi)-\frac{1}{V} \int_{X} \varphi \omega^{n}
$$

where the functional $J$ is given by

$$
J_{\omega}(\varphi)=\frac{\sqrt{-1}}{2 \pi V} \cdot \frac{1}{n+1} \sum_{i=0}^{n-1}(i+1) \partial \varphi \wedge \bar{\partial} \varphi \wedge \omega_{\varphi}^{n-i-1} \wedge \omega^{i}
$$

The variational derivative of the functional $J_{\omega}(\varphi)$ is well-known (c.f. [T3]). For a smoothly varying family of potentials $\varphi(t), t \in(-\epsilon, \epsilon)$, we have

$$
-\frac{d}{d t} F_{\omega}^{0}(\varphi(t))=\frac{1}{V} \int_{X} \dot{\varphi}(t) \omega_{\varphi}^{n}
$$

Henceforth we fix $\omega=\omega_{F S}=\frac{\sqrt{-1}}{2 \pi} \partial \bar{\partial} \log |x|^{2}$ to be the Fubini-Study Kähler form on $X$. For $\sigma \in G$ let

$$
\varphi_{\sigma}=\log \left(\frac{|\sigma x|^{2}}{|x|^{2}}\right)=\log \left(\frac{{ }^{t} \bar{x}^{t} \bar{\sigma} \sigma x}{|x|^{2}}\right)=\log \left(\frac{x^{*} \sigma^{*} \sigma x}{x^{*} x}\right)
$$

Then

$$
\sigma^{*} \omega=\omega+\frac{\sqrt{-1}}{2 \pi} \partial \bar{\partial} \varphi_{\sigma} \equiv \omega_{\varphi_{\sigma}} \equiv \omega_{\sigma} .
$$

Let $c$ be a traceless hermitian matrix, $\sigma(t)=\exp (c t) \sigma_{0}$, and $F\left(\sigma_{0}, c, t\right)=$ $F_{\omega}^{0}\left(\varphi_{\sigma(t)}\right)$. We say that $\sigma_{0}$ is a critical point of $F_{\omega}^{0}$ if and only if $\left.F^{\prime}\left(\sigma_{0}, c, t\right)\right|_{t=0}=0$ for all traceless hermitian $c$. Note that

$$
\frac{d}{d t} \varphi_{\sigma(t)}=\dot{\varphi}_{\sigma}=\frac{x^{*} \sigma^{*}\left(c^{*}+c\right) \sigma x}{x^{*} \sigma^{*} \sigma x}
$$

\section{Lemma 3.1}

1. A matrix $\sigma_{0}$ is a critical point of $F^{0}$ if and only if the manifold $\sigma_{0}(X)$ is balanced.

2. For all $\sigma_{0}, c$ and $t$ we have the formula

$$
-F^{\prime}\left(\sigma_{0}, c, t\right)=\frac{1}{V} \int_{X} \frac{x^{*} \sigma^{*}\left(c^{*}+c\right) \sigma x}{x^{*} \sigma^{*} \sigma x} \omega_{\varphi}^{n}
$$

3. For all $\sigma_{0}, c$ and $t$ we have $-F^{\prime \prime}\left(\sigma_{0}, c, t\right) \geq 0$. In fact we have

$$
-V F^{\prime \prime}\left(\sigma_{0}, c, t\right)=\frac{1}{n !} \int_{X} \iota_{\tilde{c}}\left(\omega_{\varphi}^{n+1}\right)
$$


where $\tilde{c}$ is the vector field on $\mathbf{P}^{N}$ generated by the infinitesimal action of $\exp (c t)$.

Remark. The only non-trivial part of the lemma is part 3. Zhang [Z] also proves that $-F^{\prime \prime}\left(\sigma_{0}, c, t\right) \geq 0$, by making use of a result of Deligne [D]. Another proof was given by Tian [T4]. We give a more elementary proof by a direct calculation.

Proof of Lemma 3.1. Part two follows immediately from (3.2) and (3.3a), and part one follows from part two. To prove part 3 , we differentiate both sides of (3.4):

$$
\begin{aligned}
-V F^{\prime \prime}=\int_{X} & \left\{\frac{\left(x^{*} \sigma^{*} \sigma x\right)\left(x^{*} \sigma^{*}\left[c^{*}\left(c^{*}+c\right)+\left(c^{*}+c\right) c\right] \sigma x\right)-\left(x^{*} \sigma^{*}\left(c^{*}+c\right) \sigma x\right)^{2}}{\left(x^{*} \sigma^{*} \sigma x\right)^{2}} \omega_{\varphi}^{n}\right. \\
& \left.-n \partial\left(\frac{x^{*} \sigma^{*}\left(c^{*}+c\right) \sigma x}{x^{*} \sigma^{*} \sigma x}\right) \wedge \bar{\partial}\left(\frac{x^{*} \sigma^{*}\left(c^{*}+c\right) \sigma x}{x^{*} \sigma^{*} \sigma x}\right) \omega_{\varphi}^{n-1}\right\}
\end{aligned}
$$

We may assume that $c$ is a traceless diagonal matrix with real entries. As before, we write $u=2 c$, and we let $u_{0}, \ldots, u_{N}$ be the diagonal entries of $U$. Since we can view $\mathbf{P}^{N}$ as the set of elements in $\mathbf{P}^{N+1}$ whose first entry is zero, we may also assume (after replacing $N$ by $N+1$ ) that $u_{0}=0$.

We may assume that $\sigma=I$ so that $\omega=\omega_{F S}$. Recall that on the coordinate chart $U_{0}=\left\{\left(1, z_{1}, \ldots, z_{N}\right) \subseteq \mathbf{C P}^{N}\right\}$, the Fubini-Study metric is given by:

$$
\omega=\frac{d z_{i} \wedge d \bar{z}_{i}}{1+|z|^{2}}-\frac{\bar{z}_{i} d z_{i} \wedge z_{j} d \bar{z}_{j}}{\left(1+|z|^{2}\right)^{2}}
$$

Thus if $A=\sum_{i=1}^{N} a_{i} \partial_{z_{i}}$ is a tangent vector at the point $x=\left(1, z_{1}, \ldots, z_{N}\right)$, then

$$
\omega(x)(A, \bar{A})=\frac{x^{*} x\left(\left|a_{1}\right|^{2}+\cdots+\left|a_{N}\right|^{2}\right)-\left|a_{1} \bar{z}_{1}+\cdots+a_{N} \bar{z}_{N}\right|^{2}}{\left(x^{*} x\right)^{2}}
$$

At $\sigma=I$ and $\omega=\omega_{F S}$, the first term in the integrand on the right side of (3.5) is then immediately seen to coincide with $\omega(x)\left(A_{0}, \bar{A}_{0}\right)$, where $A_{0}$ is defined to be the vector $A_{0}=\left(u_{1} z_{1}, \cdots, u_{N} z_{N}\right)$. Similarly, an explicit calculation gives

$$
\partial\left(\frac{x^{*} u x}{x^{*} x}\right)(A)=\frac{1}{\left(x^{*} x\right)^{2}}\left(x^{*} x\left(A \mid \bar{A}_{0}\right)-(A \mid \bar{z})\left(z \mid \bar{A}_{0}\right)\right)=\omega(x)\left(A, \bar{A}_{0}\right)
$$


Let $A_{1}, \cdots, A_{n}$ be now $n$ arbitrary tangent vectors at the point $x$. Then, by the definition of the wedge product,

$$
\begin{aligned}
\left(\partial\left(\frac{x^{*} u x}{x^{*} x}\right)\right. & \left.\wedge \bar{\partial}\left(\frac{x^{*} u x}{x^{*} x}\right) \wedge \omega^{n-1}\right)\left(A_{1}, \cdots, A_{n}\right) \\
& =\sum_{j, k=1}^{n}(-1)^{j+k} \omega\left(A_{j}, \bar{A}_{0}\right) \omega\left(A_{0}, \bar{A}_{k}\right) \omega^{n-1}\left(\left\{A_{p}\right\}_{p \neq j},\left\{\bar{A}_{n}\right\}_{q \neq k}\right)
\end{aligned}
$$

But wedge powers of a $(1,1)$-form are given by

$$
\omega^{K}\left(A_{1}, \cdots, A_{K}, \bar{A}_{1}, \cdots, \bar{A}_{K}\right)=K ! \operatorname{det} \omega\left(A_{j}, \bar{A}_{k}\right)
$$

Substituting this in the full integrand on the right hand side of (3.5), we obtain

$$
\begin{gathered}
n !\left(\omega\left(A_{0}, \bar{A}_{0}\right) \operatorname{det}_{1 \leq p, q \leq n} \omega\left(A_{p}, \bar{A}_{q}\right)\right. \\
\left.+\sum_{j, k=1}^{n}(-1)^{j+k+1} \omega\left(A_{j}, \bar{A}_{0}\right) \omega\left(A_{0}, \bar{A}_{k}\right) \operatorname{det}_{\substack{p \neq j \\
q \neq k}} \omega\left(A_{p}, \bar{A}_{q}\right)\right)
\end{gathered}
$$

The expression between parentheses can be recognized as the expansion along the first row (or the first column) of the determinant of the $(n+$ $1) \times(n+1)$ matrix $\omega\left(A_{p}, \bar{A}_{q}\right), 0 \leq p, q \leq n$. Since $A_{0}$ is readily recognized as the vector field $\tilde{c}$, Part 3 of Lemma 3.1 follows. Q.E.D.

Proof of Theorem 4. Once Theorem 5 is available, Theorem 4 can be proved in exactly the same manner as Theorem 3. Q.E.D.

\section{The $F_{\omega}^{0}$ functional and the Chow point.}

Fix positive integers $n<N$ and let $G r\left(N-n-1, \mathbf{P}^{N}\right)$ be the space of $N-n-1$ projective planes in $\mathbf{P}^{N}$. Then $G r\left(N-n-1, \mathbf{P}^{N}\right)=G r\left(N-n, \mathbf{C}^{N+1}\right)$, the set of $N-n$ vector subspaces of $\mathbf{C}^{N+1}$. Note that $G=S L(N+1, \mathbf{C})$ acts on $G r$ in a natural way. Recall the notation introduced in $\S 1$, namely $P \ell: G r(N-$ $\left.n-1, \mathbf{P}^{N}\right) \rightarrow \mathbf{P}\left(\Lambda^{N-n} \mathbf{C}^{N+1}\right)$ is the Plücker embedding, $O(1)=P \ell^{*} H$, where $H$ is the hyperplane bundle on $\mathbf{P}\left(\Lambda^{N-n} \mathbf{C}^{N+1}\right)$, and $\omega_{G r}=P l^{*} \Omega_{F S}$ where $\Omega_{F S}$ is the Fubini-Study metric on $\mathbf{P}\left(\Lambda^{N-n} \mathbf{C}^{N+1}\right)$.

Now let $d$ be a positive integer and define a norm on the vector space $H^{0}(G r, O(d))$ as follows: If $f \in H^{0}(G r, O(d))$ then

$$
\log \|f\|^{2}=\frac{1}{D} \int_{G r} \log \frac{|f(z)|^{2}}{|P l(z)|^{2 d}} \omega_{G r}^{m}
$$

where $D=\int_{G r} \omega_{G r}^{m+1}$ and $m+1=(N-n)(n+1)$ is the dimension of $G r$. 
Theorem 5. Let $X \subseteq \mathbf{P}^{N}$ be a smooth projective variety of dimension $n$. Let $\omega$ be the Fubini-Study metric on $X$ and let $f=\operatorname{Chow}(X)$ be the Chow point of $X$. Then

$$
-V(n+1) F_{\omega}^{0}\left(\varphi_{\sigma}\right)=\log \frac{\|\sigma \cdot \operatorname{Chow}(X)\|^{2}}{\|\operatorname{Chow}(X)\|^{2}}
$$

This theorem is equivalent to one proved by Zhang in [Z], using Deligne pairings. Paul $[\mathrm{P}]$ shows, using a different method, that the difference of the left and right sides of (4.1) is a bounded continuous function on $G$. He also shows how such estimates can be applied to give a new proof of Mumford's theorem on the stability of curves. We shall give a different proof of their theorem, in the spirit of the proof of Theorem 1.

Proof. Let $\Gamma=\left\{(x, z) \in \mathbf{P}^{N} \times G r: x \in z\right\}$ and let $\pi_{i}$ be the projection map of $\Gamma$ onto the two factors. We make use of the following formula:

$$
\pi_{1 *} \pi_{2}^{*} \omega_{G r}^{m+1}=D \omega^{n+1}
$$

Since both sides are invariant under the $U(N+1)$ action, they are equal up to a constant. The constant is the ratio of $\int_{G r} \omega_{G r}^{m+1}$ and $\int_{\mathbf{P}^{n+1}} \omega^{n+1}$, which is equal to $D$ by definition.

Now let $\sigma(t)$ be a path in $G$, and set $f^{\sigma}(z)=f\left(\sigma^{-1}(z)\right)$. We compute

$$
\begin{aligned}
\frac{d}{d t} \log \left\|f^{\sigma}\right\|^{2} & =\frac{1}{D} \frac{d}{d t} \int_{G r} \log \frac{\left|f\left(\sigma^{-1} z\right)\right|^{2}}{|P l(z)|^{2 d}} \omega_{G r}^{m+1} \\
& =\frac{1}{D} \frac{d}{d t} \int_{G r} \log \frac{|f(z)|^{2}}{|P l(\sigma z)|^{2 d}}\left(\sigma^{*} \omega_{G r}\right)^{m+1} \\
& =\frac{1}{D} \frac{d}{d t} \int_{G r} \log \frac{|f(z)|^{2}}{|P l(z)|^{2 d}}\left(\sigma^{*} \omega_{G r}\right)^{m+1}-\frac{d}{D} \frac{d}{d t} \int_{G r} \Phi_{\sigma}\left(\sigma^{*} \omega_{G r}\right)^{m+1} \\
& \equiv A-B
\end{aligned}
$$

where $\Phi_{\sigma}=\log \mid P l\left(\left.\sigma(z)\right|^{2} /|P l(z)|^{2}\right.$. Since $\sigma^{*} \omega_{G r}=\omega_{G r}+\frac{\sqrt{-1}}{2 \pi} \partial \bar{\partial} \Phi_{\sigma}$, we obtain, using the Poincare-Lelong formula

$$
\begin{aligned}
A & =\frac{1}{D} \int_{G r} \log \frac{|f(z)|^{2}}{|P l(z)|^{2 d}}(m+1)\left(\sigma^{*} \omega_{G r}\right)^{m} \frac{\sqrt{-1}}{2 \pi} \partial \bar{\partial} \dot{\Phi}_{\sigma} \\
& =\frac{1}{D} \int_{Z} \dot{\Phi}_{\sigma}(m+1)\left(\sigma^{*} \omega_{G r}\right)^{m}-\frac{d}{D} \int_{G r} \dot{\Phi}_{\sigma}(m+1)\left(\sigma^{*} \omega_{G r}\right)^{m} \omega_{G r} \\
B & =\frac{d}{D} \int_{G r} \dot{\Phi}_{\sigma}\left(\sigma^{*} \omega_{G r}\right)^{m+1}+\frac{d}{D} \int_{G r} \Phi_{\sigma} \frac{\sqrt{-1}}{2 \pi} \partial \bar{\partial} \dot{\Phi}_{\sigma}(m+1)\left(\sigma^{*} \omega_{G r}\right)^{m}
\end{aligned}
$$


We integrate by parts in the second term in $B$. Combining the result with the other term in $B$ and the second term in $A$, we can rewrite the above equation as

$$
\frac{d}{d t} \log \left\|f^{\sigma}\right\|^{2}=\frac{1}{D} \int_{Z} \dot{\Phi}_{\sigma}(m+1)\left(\sigma^{*} \omega_{G r}\right)^{m}-\frac{d(m+2)}{D} \int_{G r} \dot{\Phi}_{\sigma}\left(\sigma^{*} \omega_{G r}\right)^{m+1}
$$

After making a change a variables $z \mapsto \sigma^{-1} z$, we may assume that the $\sigma$ in the second integral is the identity. We conclude that the second integral is zero since the hyperplane bundle over the Grassmannian variety is balanced. More simply, we may write $\dot{\Phi}_{\sigma}(d)=\operatorname{tr}\left(Z^{*} d Z\right)$ with $Z^{*} Z=1$ and $d=c+c^{*}$, $\operatorname{tr}(d)=0$. Then the integral

$$
M(d)=\int_{G r} \operatorname{tr}\left(Z^{*} d Z\right) \omega_{G r}^{m+1}
$$

satisfies $M\left(u^{*} d u\right)=M(d), M\left(d_{1}+d_{2}\right)=M\left(d_{1}\right)+M\left(d_{2}\right)$. The first property allows us to assume that $d$ is diagonal. The second implies that $M(d)$ is the same if we average it over the permutations of the eigenvalues of $d$. Thus we must have

$$
M(d)=0 .
$$

It remains to show that the integral over $Z$ in (4.4) can be reexpressed as an integral over $X$. For this, we apply $\sigma(t)=\exp (c t) \sigma_{0}$ to both sides of (4.2), differentiate with respect to $t$ and evaluate at $t=0$ :

$$
\pi_{1 *} \pi_{2}^{*}\left((m+1) \sigma^{*} \omega_{G r}^{m} \partial \bar{\partial} \dot{\Phi}_{\sigma_{0}, c}\right)=D(n+1)\left(\sigma^{*} \omega^{n}\right) \partial \bar{\partial} \dot{\varphi}_{\sigma_{0}, c}
$$

where $\Phi_{\sigma_{0}, c}\left(\right.$ resp. $\left.\varphi_{\sigma_{0}, c}\right)$ is the derivative of $\Phi_{\sigma(t)}\left(\operatorname{resp} . \varphi_{\sigma(t)}\right)$ at $t=0$. This shows that

$$
\pi_{1 *} \pi_{2}^{*}\left(\dot{\Phi}_{\sigma_{0}, c}(m+1)\left(\sigma_{0}^{*} \omega_{G r}^{m}\right)\right)=D(n+1) \dot{\varphi}_{\sigma_{0}, c}\left(\sigma_{0}^{*} \omega^{n}\right)+\eta\left(\sigma_{0}, h\right)
$$

for some closed smooth form $\eta\left(\sigma_{0}, h\right)$ where $h=c+c^{*}$ is traceless hermitian. We claim that $\eta\left(\sigma_{0}, h\right)$ is exact. Note the following properties: $\eta\left(\sigma_{0}, u^{*} h u\right)=$ $\eta\left(u \sigma_{0}, h\right)$ for all unitary matrices $u$. Also, $\eta\left(\sigma_{0}, h\right)=\sigma_{0}^{*}(\eta(I, h))$ where $I$ is the identity matrix, and $\eta\left(\sigma_{0}, h\right)$ is a linear function of $h$. This shows that we may assume that $\sigma_{0}=I$ and $h$ is diagonal with real eigenvalues whose sum is zero. In fact, using the linearity property we may assume that $h$ is the matrix whose diagonal entries are $(1,-1,0, \ldots, 0)$. Now let $\iota: \mathbf{P}^{n} \hookrightarrow \mathbf{P}^{N}$ be the map $\left(x_{0}, \ldots, x_{n}\right) \mapsto\left(x_{0}, \ldots, x_{n}, 0,0, \ldots, 0\right)$ and let $X^{\prime}$ denote the image of $\iota$. To show that $\eta(I, h)$ is exact, it suffices to show that its integral over $X^{\prime}$ is zero. 
To see this, first observe that $\int_{X^{\prime}} \dot{\varphi}_{I, h} \omega^{n}=\operatorname{tr}(h M)$ where $M=\int_{X^{\prime}} x x^{*} \omega^{n}$ and $x$ is the vector of homogeneous coordinates, normalized to have length one (this follows form (1.2)). Let $U_{n} \subseteq U(N+1)$ be the group of unitary matrices which map $X^{\prime}$ into itself. Then $M$ commutes with $U_{n}$ which means that it's a diagonal matrix whose first $n+1$ entries are all equal. But this means that $\operatorname{tr}(h M)=0$ and thus $\int_{X^{\prime}} \dot{\varphi}_{I, h} \omega^{n}=0$. Similarly, $\int_{Z^{\prime}} \dot{\Phi}_{I, h} \omega_{G r}^{m}=0$ where $Z^{\prime} \subseteq G r$ is the zero locus of the Chow point of $X^{\prime}$. This implies that $\int_{X^{\prime}} \eta(I, h)=0-0=0$, which proves our claim.

Plugging this into (4.4) we obtain:

$$
\frac{d}{d t} \log \left\|f^{\sigma}\right\|^{2}=\int_{X}(n+1) \dot{\varphi}_{\sigma}\left(\sigma^{*} \omega^{n}\right)
$$

Comparing this with the derivative of $F_{\omega}^{0}$ given in (3.2), we obtain Theorem 5. Q.E.D.

Remark. A similar argument shows that for every $k$ such that $0 \leq k \leq n$, there is a constant $D_{k}>0$ such that

$$
\begin{gathered}
\pi_{1 *} \pi_{2}^{*} \omega_{G r}^{m-k}=D_{k} \cdot \omega^{n-k} \\
\pi_{1 *} \pi_{2}^{*}\left(\dot{\Phi}_{\sigma_{0}, c}(m+1-k)\left(\sigma_{0}^{*} \omega_{G r}^{m-k}\right)\right)=D_{k}(n+1-k) \dot{\varphi}_{\sigma_{0}, c}\left(\sigma_{0}^{*} \omega^{n-k}\right)+\eta_{k}\left(\sigma_{0}, h\right)
\end{gathered}
$$

where $\eta_{k}\left(\sigma_{0}, h\right)$ is a smooth exact $(n-k, n-k)$ form.

\section{The Mabuchi energy: Hypersurface Case.}

We turn now to the setting of main interest in the present paper, namely that of Theorem 1. In this section, we discuss first the case of hypersurfaces. In this case, our construction of $\|\cdot\|_{\#}$ shares many features with Tian's construction of $\|\cdot\|_{Q}$ in [T1]. However, the explicit formulas in our approach will facilitate the generalization to higher codimension. We present our proof of the hypersurface case in such a way as to apply verbatim to the higher codimension case as much as possible, so that the difficulties inherent to this latter case will be more transparent in the next section.

We first recall the definition of the Mabuchi energy [M]. Let $X$ be a Kähler manifold, with Kähler form $\omega$. Then the Mabuchi energy $\nu_{\omega}(\varphi)$ is defined for all Kähler forms $\omega_{\varphi}=\omega+\frac{\sqrt{-1}}{2 \pi} \partial \bar{\partial} \varphi$ by choosing a path $\omega_{t}=\omega+\frac{\sqrt{-1}}{2 \pi} \partial \bar{\partial} \varphi_{t}$, 
$0 \leq t \leq 1, \omega_{0}=\omega, \omega_{1}=\omega$, and setting

$$
\begin{aligned}
\nu_{\omega}(\varphi) & =-\frac{1}{\operatorname{vol}(X)} \int_{0}^{1} \int_{X} \dot{\varphi}_{t}\left(s\left(\omega_{t}\right)-\mu\right) \omega_{t}^{n} d t \\
& =-\frac{1}{\operatorname{vol}(X)} \int_{0}^{1} \int_{X} \dot{\varphi}_{t}\left(n \operatorname{Ric}\left(\omega_{t}\right)-\mu \omega_{t}\right) \omega_{t}^{n-1} d t
\end{aligned}
$$

where the function $s\left(\omega_{t}\right)$ is the scalar curvature of $\omega_{t}$, and $\mu=n(n+2-d)$ is the average scalar curvature. The Ricci curvature is defined by $\operatorname{Ric}(\omega)=$ $-\frac{\sqrt{-1}}{2 \pi} \partial \bar{\partial} \log \left(\omega^{n}\right)$. Thus if we view $\omega^{n}$ as a metric $h$ on the canonical bundle $K=\Lambda^{n} T_{X}^{*}$, and then the Ricci curvature is just the curvature $-\frac{\sqrt{-1}}{2 \pi} \partial \bar{\partial} \log h$ of this metric.

There is another more direct way of defining the Mabuchi energy, pointed out by Tian [T1] and Chen [C1], which does not require an integral along paths:

$$
\nu_{\omega}(\varphi)=\frac{1}{\operatorname{vol}(X)} \int_{X}\left(\log \left(\frac{\omega_{\varphi}^{n}}{\omega^{n}}\right) \omega_{\varphi}^{n}+h_{\omega}\left(\omega^{n}-\omega_{\varphi}^{n}\right)\right)-\frac{1}{n}\left(I_{\omega}(\varphi)-J_{\omega}(\varphi)\right)
$$

where $\omega_{\varphi}=\omega+\frac{\sqrt{-1}}{2 \pi} \partial \bar{\partial} \varphi, \operatorname{vol}(X)=\int_{X} \omega^{n}$ is the volume of $X, J_{\omega}(\varphi)$ is the functional introduced before, and $I_{\omega}(\varphi)=\frac{1}{\operatorname{vol}(X)} \int_{X} \varphi\left(\omega^{n}-\omega_{\varphi}^{n}\right)$ is the other functional introduced by Yau [Y1] and Aubin. In [Z], Zhang used the Deligne pairing $<\mathcal{L}_{0}, \mathcal{L}_{1}, \cdots, \mathcal{L}_{n}>_{(X / S)}$ with $\mathcal{L}_{i}=O(1)$ for all $0 \leq i \leq n$ to obtain the functional $F_{\omega}^{0}$. Here $\pi: X \rightarrow S$ is a flat projective morphism of integral schemes of relative dimension $n$, so that each fiber $X_{s}, s \in S$ is a projective variety of dimension $n$, and $\mathcal{L}_{i}$ are line bundles over $X$. If we choose instead $\mathcal{L}_{i}=O(1)$ for $0 \leq i<n, \mathcal{L}_{n}=\mathcal{K}$, then we obtain another expression for the Mabuchi functional which does not require a path integration

$$
\nu_{\omega}(\varphi)=-E_{\omega}(\varphi)-\mu \operatorname{vol}(X) F_{\omega}^{0}(\varphi)
$$

Here the functional $E(\varphi)$ is defined by

$$
\begin{aligned}
<O(1) \otimes & \mathcal{O}(\varphi), \cdots, O(1) \otimes \mathcal{O}(\varphi), \mathcal{K} \otimes \mathcal{O}\left(\log \frac{\omega_{\varphi}^{n}}{\omega^{n}}\right)>_{X / S} \\
& =<O(1), \cdots, O(1), \mathcal{K}>_{X / S} \otimes \mathcal{O}(E)
\end{aligned}
$$

For our purposes, it is most convenient to observe that $F_{\omega}^{0}$ can be rewritten in the form proposed by Futaki $[\mathrm{F}]$

$$
F_{\omega}^{0}(\varphi)=-\frac{1}{\operatorname{vol}(X)} \frac{1}{n+1} \int_{X} \varphi \sum_{i=0}^{n} \omega^{i} \omega_{\varphi}^{n-i}
$$


and to recast the Mabuchi energy $\nu_{\omega}(\varphi)$ in the following form

$$
\begin{aligned}
\nu_{\omega}(\varphi)= & \frac{1}{\operatorname{vol}(X)} \int_{X}\left\{\log \left(\frac{\omega_{\varphi}^{n}}{\omega^{n}}\right) \omega_{\varphi}^{n}\right. \\
& \left.-\varphi\left(\operatorname{Ric}(\omega) \sum_{i=0}^{n-1} \omega^{i} \omega_{\varphi}^{n-1-i}-\frac{\mu(X)}{n+1} \sum_{i=0}^{n} \omega^{i} \omega_{\varphi}^{n-i}\right)\right\}
\end{aligned}
$$

where $\mu(X)$, the average of the scalar curvature of $\omega_{X}$, is given by the following formula:

$$
\mu(X)=n(n+2-d)
$$

Here we use $\operatorname{vol}(X)$ to distinguish the volume of $X$ from the volume $V=$ $\operatorname{vol}(Z)$ of the Chow variety $Z$, which also enters our formulas.

Consider now the case of a smooth hypersurface $X \subset \mathbf{P}^{n+1}$. In this case, $X$ coincides with its Chow variety $Z, n=m$, the Grassmannian $G r$ reduces to $\mathbf{P}^{m+1}=\mathbf{P}^{n+1}, \operatorname{vol}(X)=\operatorname{vol}(Z)=V$, and the functions $\Phi_{\sigma}$ and $\varphi_{\sigma}$ coincide.

Theorem 1 simplifies considerably, and we restate it as follows

Theorem 6. Let $Z \subseteq \mathbf{P}^{m+1}$ be a smooth hypersurface of degree $d$ and let $f$ be the section of $H^{0}\left(\mathbf{P}^{m+1}, O(d)\right)$ which defines $Z$. Let $\omega_{F S}$ be the Fubini-Study metric on $\mathbf{P}^{m+1}$, and let $\omega$ be the restriction of $\omega_{F S}$ to $Z$. For $\sigma \in S L(m+2, \mathbf{C})$ we have

$$
\nu_{\omega}\left(\Phi_{\sigma}\right)=\frac{D(m+2)(d-1)}{V(m+1)} \log \frac{\|\sigma \cdot f\|_{\#}^{2}}{\|f\|_{\#}^{2}}
$$

Proof. We evaluate first the contributions of the logarithmic terms from $\|f\|_{\#}$ on the right side of (5.4)

$$
\frac{1}{V} \cdot \int_{Z} \log \left(\frac{\sigma^{*} \omega^{m} \wedge \partial \bar{\partial} \frac{|f(z)|^{2}}{|\sigma z|^{2}}}{\sigma^{*} \omega^{m+1}}\right) \sigma^{*} \omega^{m}-\frac{1}{V} \cdot \int_{Z} \log \left(\frac{\omega^{m} \wedge \partial \bar{\partial} \frac{|f(z)|^{2}}{|z|^{2}}}{\omega^{m+1}}\right) \omega^{m}
$$

Writing $\omega^{m}=\left(\omega^{m}-\sigma^{*} \omega^{m}\right)+\sigma^{*} \omega^{m}$ in the second integral, the right side of (5.5) becomes:

$$
\begin{aligned}
\int_{Z} \log & \left(\frac{\sigma^{*} \omega^{m} \wedge \partial \bar{\partial} \frac{|f(z)|^{2}}{|\sigma z|^{2}}}{\omega^{m} \wedge \partial \bar{\partial} \frac{|f(z)|^{2}}{|z|^{2}}} \cdot \frac{\omega^{m+1}}{\sigma^{*} \omega^{m+1}}\right) \sigma^{*} \omega^{m} \\
& -\int_{Z} \log \left(\frac{\omega^{m} \wedge \partial \bar{\partial} \frac{|f(z)|^{2}}{|z|^{2}}}{\omega^{m+1}}\right)\left(-\frac{\sqrt{-1}}{2 \pi} \partial \bar{\partial} \Phi_{\sigma}\right) \sum_{i=0}^{m-1} \omega^{m-i} \sigma^{*} \omega^{i} \equiv A-B
\end{aligned}
$$


To evaluate the integrands in the last equation, we choose $(m+1)$ linearly independent holomorphic vector fields $X_{1}, \ldots X_{m}, Y$ in an open neighborhood of a point $z \in X \subseteq \mathbf{P}^{m+1}$ in such a way that $X_{1}, \ldots, X_{m}$ are tangent to $Z$. Using the definition of the wedge product, we evaluate

$$
\begin{gathered}
\left(\sigma^{*} \omega^{m} \wedge \partial \bar{\partial}\left(\frac{|f(z)|^{2}}{|\sigma z|^{2 d}}\right)\right)\left(X_{1}, X_{2}, \ldots, X_{m}, Y\right)= \\
\sum_{\pi} \sigma^{*}(\omega) \otimes \cdots \sigma^{*}(\omega) \otimes \partial \bar{\partial}\left(\frac{|f(z)|^{2}}{|\sigma z|^{2 d}}\right)\left(X_{1} \otimes \bar{X}_{1} \otimes X_{2} \otimes \bar{X}_{2} \cdots X_{m} \otimes \bar{X}_{m}, Y \otimes \bar{Y}\right)^{\pi}
\end{gathered}
$$

where $\pi$ ranges over all the permutations $\pi$ of the sequence $\left(X_{1}, \bar{X}_{1}, \ldots, X_{m}, \bar{X}_{m}, Y, \bar{Y}\right)$. Since $f$ vanishes on $Z$, the only terms in the sum which are non-zero are those corresponding to permutations which permute the $X_{i}$ and the $\bar{X}_{i}$, but fix $Y$ and $\bar{Y}$. Furthermore, note that when we apply $\partial \bar{\partial}$ to $\left(\frac{|f(z)|^{2}}{|\sigma z|^{2 d}}\right)$, the product rule will yield several terms. But when we restrict to $X$, the only term which doesn't vanish is the one where the $\partial \bar{\partial}$ lands on $|f(z)|^{2}$ (again, since $f$ vanishes on $Z$ ). Thus

$$
\left.\partial \bar{\partial}\left(\frac{|f(z)|^{2}}{|\sigma z|^{2 d}}\right)(Y \otimes \bar{Y})\right|_{Z}=\frac{1}{|\sigma z|^{2 d}} \cdot|Y(f)|^{2}
$$

and we may write

$$
\begin{aligned}
A & =\int_{Z} \log \left(\frac{\sigma^{*} \omega^{m}\left(X_{1}, \ldots, X_{m}\right) \cdot \frac{|Y(f)|^{2}}{|\sigma z|^{2 d}}}{\omega^{m}\left(X_{1}, \ldots, X_{m}\right) \cdot \frac{|Y(f)|^{2}}{|z|^{2 d}}} \cdot \frac{\omega^{m+1}}{\sigma^{*} \omega^{m+1}}\right) \sigma^{*} \omega^{m} \\
B & =\int_{Z} \log \left(\frac{\omega^{m}\left(X_{1}, \ldots, X_{m}\right) \cdot \frac{|Y(f)|^{2}}{|z|^{2 d}}}{\omega^{m+1}\left(X_{1}, \ldots, X_{m}, Y\right)}\right)\left(-\frac{\sqrt{-1}}{2 \pi} \partial \bar{\partial} \Phi_{\sigma}\right) \eta_{\sigma}
\end{aligned}
$$

Cancelling the common factor $|Y(f)|^{2}$ in $A$, and using the simple fact that $\log \frac{\sigma^{*} \omega^{m+1}}{\omega^{m+1}}=-(m+2) \Phi_{\sigma}$, we obtain

$$
A=\int_{Z}\left\{\log \left(\frac{\sigma^{*} \omega^{m}}{\omega^{m}}\right)+(m+2-d) \Phi_{\sigma}\right\} \cdot \sigma^{*} \omega^{m}
$$

To evaluate $B$, we integrate by parts. Since $Z$ is smooth, we have

$$
B=\int_{Z}-\frac{\sqrt{-1}}{2 \pi} \partial \bar{\partial} \log \left(\frac{\omega^{m}\left(X_{1}, \ldots, X_{m}\right) \cdot \frac{|Y(f)|^{2}}{|z|^{2 d}}}{\omega^{m+1}\left(X_{1}, \ldots, X_{m}, Y\right)}\right) \Phi_{\sigma} \sum_{i=0}^{m-1} \omega^{i} \omega_{\sigma}^{m-1-i}
$$


Now we clearly have

$$
-\frac{\sqrt{-1}}{2 \pi} \partial_{X} \bar{\partial}_{X} \log \left\{\frac{|Y(f)|^{2}}{|z|^{2 d}}\right\}=d \cdot \omega
$$

since $Y$ is a transversal holomorphic vector field and $Y(f)$ is a non-vanishing holomorphic function on $Z$. Also $-\frac{\sqrt{-1}}{2 \pi} \partial \bar{\partial} \log \omega^{m+1}=(m+2) \omega$ and $-\frac{\sqrt{-1}}{2 \pi} \partial_{X} \bar{\partial}_{X} \log \left(\sigma^{*} \omega^{m}\right)=\operatorname{Ric}\left(\sigma^{*} \omega^{m}\right)$ by definition. Thus

$$
B=\int_{Z} \Phi_{\sigma}(\operatorname{Ric}(\omega)+d \omega-(m+2) \omega) \cdot \sum_{i=0}^{m-1} \omega^{i} \omega_{\sigma}^{m-1-i}
$$

This calculation of the $B$ term is very similar to a curvature calculation in Tian [T1] (see also $\mathrm{Lu}[\mathrm{Lu} 2]$ ). Finally, the remaining contributions from $\log \frac{\|\sigma \cdot \operatorname{Chow}(X)\|_{\#}^{2}}{\|\operatorname{Chow}(X)\|_{\#}^{2}}$ only involve terms of the form $\frac{\|\sigma \cdot \operatorname{Chow}(X)\|^{2}}{\|\operatorname{Chow}(X)\|^{2}}$, which are known from Theorem 5

$$
-\frac{D}{V(m+1)} \log \frac{\|\sigma \cdot \operatorname{Chow}(X)\|^{2}}{\|\operatorname{Chow}(X)\|^{2}}=F_{\omega}^{0}\left(\Phi_{\sigma}\right)=-\frac{1}{V(m+1)} \int_{Z} \Phi_{\sigma} \sum_{i=0}^{m-1} \omega^{i} \omega_{\sigma}^{m-1-i}
$$

Assembling all terms gives Theorem 6. Q.E.D.

\section{The Mabuchi energy: General Case.}

In this section we establish Theorem 1 for arbitrary codimension. Our notation is the one introduced in $\S 1$.

Let $X \subseteq \mathbf{P}^{N}$ be a smooth variety of dimension $n$ and let $Z \subseteq G r$ be the corresponding Chow variety, and $f \in H^{0}(G r, O(d))$ be a defining section for $Z$. Then $Z$ is a singular variety. We say that the embedding $X \subseteq \mathbf{P}^{N}$ is generic if it satisfies the following conditions:

1. If $Z_{s} \subseteq Z$ is the singular set of $Z$, then $Z_{s}$ has codimension one.

2. There exists a subvariety $Z_{s s} \subseteq Z_{s}$ of codimension at least one (and hence $Z_{s s} \subseteq G r$ has codimension at least two) such that $Z_{s} \backslash Z_{s s}$ is a divisor with normal crossings. In other words, for all $z \in Z_{s} \backslash Z_{s s}$, there exist local coordinates $\left(z_{0}, \ldots, z_{m}\right)$, centered at $z$, such that $f\left(z_{0}, \ldots, z_{m}\right)=z_{0} z_{1}$.

3. Let $Z_{0}=Z \backslash Z_{s}$ be the set of smooth points of $Z$. Then the scalar curvature $s\left(\omega_{Z}\right)$ is $L^{1}\left(Z_{0}\right)$ with respect to the volume form $\omega_{Z}^{m}$. 
The proof consists of two parts. In the first part, the proof is identical to that for hypersurfaces in the preceding section, if we view the arguments given there as applying to the Chow variety $Z$, which is a codimension 1 subvariety of the Grassmannian. The result of this first part is to express $\log \frac{\|\sigma \cdot \operatorname{Chow}(X)\|_{\#}^{2}}{\|\operatorname{Chow}(X)\|_{\#}^{2}}$ as the generalized Mabuchi energy $\nu_{\omega_{Z}}^{\#}\left(\Phi_{\sigma}\right)$ associated to the singular variety $Z$ (see Lemma 6.1 below). The second part of the proof consists in identifying the regular part of the generalized Mabuchi functional $\nu_{\omega_{Z}}^{\#}\left(\Phi_{\sigma}\right)$ with the Mabuchi energy $\nu_{\omega_{X}}\left(\varphi_{\sigma}\right)$ associated to the projective variety $X$ itself (see Lemma 6.2).

Let $\omega=\omega_{Z}$ be the restriction of $\omega_{G r}$ to $Z_{0}$, the set of smooth points of $Z$. Then $\operatorname{Ric}\left(\omega_{Z}\right)$, the Ricci curvature of $\omega_{Z}$, is a smooth $(1,1)$ form on $Z_{0}$. Let $V=V(Z)$ be the volume of $Z$, and let $\Gamma_{X}=\{(x, z): z \in G r, x \in X \cap z\}$. Let $p_{1}: \Gamma_{X} \rightarrow X$ and $p_{2}: \Gamma_{X} \rightarrow Z$ be the projection maps, and let $Y_{s}=p_{2}^{-1}\left(Z_{s}\right) \subseteq \Gamma_{X}$ and $Y_{s s}=p_{2}^{-1}\left(Z_{s s}\right)$.

Since $\left|s\left(\omega_{Z}\right)\right|$ is in $L^{1}\left(Z_{0}\right)$, we can set $\mu(Z)=\frac{1}{V} \int_{Z_{0}} s\left(\omega_{Z}\right) \omega_{Z}^{m}$ and define the Mabuchi functional $\nu_{\omega_{Z}}\left(\Phi_{\sigma}\right)$ associated with the regular variety $Z_{0}$ as before by

$$
\begin{aligned}
\nu_{\omega_{Z}}\left(\Phi_{\sigma}\right)= & \frac{1}{V} \int_{Z_{0}}\left\{\log \left(\frac{\sigma^{*} \omega_{Z}^{m}}{\omega_{Z}^{m}}\right) \sigma^{*} \omega_{Z}^{m}\right. \\
& \left.-\Phi_{\sigma}\left(\operatorname{Ric}\left(\omega_{Z}\right) \sum_{i=0}^{m-1} \omega_{Z}^{i} \sigma^{*} \omega_{Z}^{m-1-i}-\frac{\mu(Z)}{m+1} \sum_{i=0}^{m} \omega_{Z}^{i} \sigma^{*} \omega_{Z}^{m-i}\right)\right\}
\end{aligned}
$$

Associated to the variety $Y_{s}$ is a closed current $\left[Y_{s}\right]$, supported on $Y_{s}$, which is defined by the following equation of currents on $\Gamma_{X}$ :

$$
-\frac{\sqrt{-1}}{2 \pi} \partial \bar{\partial} \log \left(\frac{\omega_{Z}^{m} \wedge \partial \bar{\partial} \frac{|f(z)|^{2}}{|P \ell(z)|^{2 d}}}{\omega_{Z}^{m+1}}\right)=\operatorname{Ric}\left(\omega_{Z}\right)-(m+2-d) \omega_{Z}-\left[Y_{s}\right]
$$

The generalized Mabuchi energy $\nu_{\omega_{Z}}^{\#}\left(\Phi_{\sigma}\right)$ associated to the singular variety $Z$ can now be defined by

$$
\begin{aligned}
\nu_{\omega_{Z}}^{\#}\left(\Phi_{\sigma}\right)= & \nu_{\omega_{Z}}\left(\Phi_{\sigma}\right)+\frac{1}{V}\left\langle\left[Y_{s}\right], \Phi_{\sigma} \sum_{i=0}^{m-1} \omega_{Z}^{i} \sigma^{*} \omega_{Z}^{m-1-i}\right\rangle \\
& -\frac{D}{V} \cdot \frac{m \operatorname{deg}\left(Y_{s}\right)}{m+1} \cdot \log \frac{\|\sigma \cdot f\|^{2}}{\|f\|^{2}}
\end{aligned}
$$


where $\|\cdot\|$ is the norm introduced in (4.1) and $\operatorname{deg}\left(Y_{s}\right)=\frac{1}{V}\left\langle\left[Y_{s}\right], \omega_{Z}^{m-1}\right\rangle$.

Lemma 6.1 Under the above hypotheses, we have

$$
\nu_{\omega_{Z}}^{\#}\left(\Phi_{\sigma}\right)=\frac{D(m+2)(d-1)}{V(m+1)} \log \frac{\|\sigma \cdot f\|_{\#}^{2}}{\|f\|_{\#}^{2}}
$$

Proof. Since the map $p_{2}: \Gamma_{X} \rightarrow Z$ is birational, we can pull back integrals over $Z$ to integrals over $\Gamma_{Z}$ and express the right side of (6.4) as $A^{\prime}-B^{\prime}+T$ where

$$
\begin{aligned}
& A^{\prime}=\frac{1}{V} \cdot \int_{\Gamma_{X}} \log \left(\frac{\sigma^{*} \omega^{m} \wedge \partial \bar{\partial} \frac{|f(z)|^{2}}{|P \ell(\sigma z)|^{2}}}{\sigma^{*} \omega^{m+1}}\right) \sigma^{*} \omega^{m} \\
& B^{\prime}=\frac{1}{V} \cdot \int_{\Gamma_{X}} \log \left(\frac{\omega^{m} \wedge \partial \bar{\partial} \frac{|f(z)|^{2}}{|P \ell(z)|^{2}}}{\omega^{m+1}}\right) \omega^{m}
\end{aligned}
$$

and

$T=(m+2-d) \cdot F_{\omega}^{0}\left(\Phi_{\sigma}\right)=-\frac{(m+2-d)}{(m+1)} \cdot \frac{1}{V} \int_{\Gamma_{X}} \Phi_{\sigma} \cdot\left(\sum_{i=0}^{m} \omega^{i} \wedge \sigma^{*} \omega^{m-i}\right)$

where we write $\omega$ for $\omega_{Z}$.

Replacing $\omega^{m}$ by $\left(\omega^{m}-\sigma^{*} \omega^{m}\right)+\sigma^{*} \omega^{m}$ in $(6.5)$, we obtain $A^{\prime}-B^{\prime}=A-B$ where

$$
\begin{aligned}
& A=\frac{1}{V} \int_{\Gamma_{X}} \log \left(\frac{\sigma^{*} \omega^{m} \wedge \partial \bar{\partial} \frac{|f(z)|^{2}}{|P \ell(\sigma z)|^{2}}}{\omega^{m} \wedge \partial \bar{\partial} \frac{|f(z)|^{2}}{|P \ell(z)|^{2}}} \cdot \frac{\omega^{m+1}}{\sigma^{*} \omega^{m+1}}\right) \sigma^{*} \omega^{m} \\
& B=\frac{1}{V} \int_{\Gamma_{X}} \log \left(\frac{\omega^{m} \wedge \partial \bar{\partial} \frac{|f(z)|^{2}}{|P \ell(z)|^{2}}}{\omega^{m+1}}\right)\left(-\frac{\sqrt{-1}}{2 \pi} \partial \bar{\partial} \Phi_{\sigma}\right) \sum_{i=0}^{m-1} \omega^{i} \wedge \sigma^{*} \omega^{m-1-i}
\end{aligned}
$$

The same argument as that used in $\S 5$ again gives

$$
A=\frac{1}{V} \int_{\Gamma_{X}}\left\{\log \left(\frac{\sigma^{*} \omega^{m}}{\omega^{m}}\right)-(m+2-d) \Phi_{\sigma}\right\} \cdot \sigma^{*} \omega^{m}
$$


In order to evaluate $B$, we integrate (6.6) by parts:

$$
B=\frac{1}{V} \int_{\Gamma_{X}} \Phi_{\sigma} \cdot\left(\operatorname{Ric}(\omega)-(m+2-d) \omega-\left[Y_{s}\right]\right) \cdot \sum_{i=0}^{m-1} \omega^{i} \wedge \sigma^{*} \omega^{m-1-i}
$$

Assembling (6.6), (6.7) and (6.8) and making use of

$$
m(m+2-d)=\frac{1}{V} \int_{\Gamma_{X}} m\left(\operatorname{Ric}(\omega)-\left[Y_{s}\right]\right) \omega^{m-1}=\mu(Z)-m \operatorname{deg}\left(Y_{s}\right)
$$

we obtain (6.4).

Remarks on $\left[Y_{s}\right]$

1. The fact that $\left[Y_{s}\right]$ is supported on $Y_{s}$ follows from (5.8).

2. It's not difficult to see that $\left[Y_{s}\right]$ is given by integration over the smooth points of the variety $Y_{s}$.

3. The current $\left[Y_{s}\right]$ is also defined by the equation of currents: $\partial \bar{\partial} \log |\nabla F|^{2}=\left[Y_{s}\right]$, where $\nabla F$ is the gradient of the holomorphic function which locally defines $Z$. To see this, we let $\xi$ be the function

$$
\xi(z)=\frac{\omega^{m} \wedge \partial \bar{\partial} \frac{|f(z)|^{2}}{|P \ell(z)|^{2}}}{\omega^{m+1}}
$$

Then $\xi$ is a smooth function on $G r$. Let $y_{0} \in \Gamma_{X}$, and choose a coordinate system of $\left(w_{0}, \ldots, w_{m}\right)$ of a neighborhood of $p_{2}\left(y_{0}\right) \in G r$. Then

$$
\xi(y)=\left(\xi \circ p_{2}\right)(y)=\sum_{i=0}^{m}\left|\frac{\partial F}{\partial w_{i}}\right|^{2}\left(p_{2}(y)\right)=\sum_{i=0}^{m}\left|f_{i}(y)\right|^{2}
$$

in some coordinate neighborhood of $y_{0} \in \Gamma_{X}$, where $F$ is an analytic function whose divisor is $Z$ and the $f_{i}(y)$ are analytic functions whose set of common zeros is precisely $Y_{s}$.

Lemma 6.2 Assume that $X \subseteq \mathbf{P}^{N}$ is generic. Then

$$
\nu_{\omega_{Z}}\left(\Phi_{\sigma}\right)=\nu_{\omega_{F S}}\left(\varphi_{\sigma}\right)
$$

Proof. As in the proof of Theorem 5, we differentiate both sides of (6.9) with respect to $t$ and show that the two sides are equal. Thus let $c$ be an 
arbitrary $(N+1) \times(N+1)$ traceless matrix, and let $\sigma(t)=\exp (c t) \sigma_{0}$ for some fixed $\sigma_{0} \in G$.

Recall that $\nu_{\omega_{Z}}\left(\Phi_{\sigma}\right)$ is given by (6.1). Let $\psi_{\sigma}=\log \left(\frac{\sigma^{*} \omega_{Z}^{m}}{\omega_{Z}^{m}}\right)$, and note that $\left(\sigma^{*} \omega\right)^{m} \cdot \frac{d}{d t} \psi_{\sigma}=\left(\sigma^{*} \omega\right)^{m} \cdot \frac{m\left(\sigma^{*} \omega\right)^{m-1} \frac{\sqrt{-1}}{2 \pi} \partial \bar{\partial} \dot{\Phi}_{\sigma}}{\left(\sigma^{*} \omega\right)^{m}}=m\left(\sigma^{*} \omega\right)^{m-1} \frac{\sqrt{-1}}{2 \pi} \partial \bar{\partial} \dot{\Phi}_{\sigma}$ so

$$
\int_{\Gamma_{X}}\left(\sigma^{*} \omega\right)^{m} \cdot \frac{d}{d t} \psi_{\sigma}=0
$$

Writing $\omega_{\sigma}=\sigma^{*} \omega$, we obtain, since $\psi_{\sigma}$ is smooth outside of $Y_{s s}$ and $Y_{s s}$ has codimension $\geq 2$

$$
\begin{aligned}
\frac{d}{d t} \int_{\Gamma_{X}} \psi_{\sigma}\left(\sigma^{*} \omega\right)^{m} & =\int_{\Gamma_{X}} \psi_{\sigma} m\left(\sigma^{*} \omega\right)^{m-1} \frac{\sqrt{-1}}{2 \pi} \partial \bar{\partial} \dot{\Phi}_{\sigma} \\
& =-\int_{\Gamma_{X}} m \dot{\Phi}_{\sigma}\left(\operatorname{Ric}\left(\omega_{\sigma}\right)-\operatorname{Ric}(\omega)\right) \omega_{\sigma}^{m-1}
\end{aligned}
$$

On the other hand,

$$
\begin{gathered}
\frac{d}{d t} \int_{\Gamma_{X}} \sum_{i=1}^{m} \Phi_{\sigma}\left(\sigma^{*} \omega\right)^{m-i} \omega^{i-1} \operatorname{Ric}(\omega)=\int_{\Gamma_{X}} \sum_{i=1}^{m} \dot{\Phi}_{\sigma}\left(\omega_{\sigma}\right)^{m-i} \omega^{i-1} \operatorname{Ric}(\omega)+ \\
\int_{\Gamma_{X}} \sum_{i=1}^{m-1} \dot{\Phi}_{\sigma}(m-i)\left(\left(\omega_{\sigma}\right)^{m-i} \omega^{i-1}-\left(\omega_{\sigma}\right)^{m-i-1} \omega^{i}\right) \operatorname{Ric}(\omega)
\end{gathered}
$$

The second term on the right hand side can be rewritten as

$$
\int_{\Gamma_{X}} \dot{\Phi}_{\sigma}\left(\sum_{i=1}^{m-1}(m-i)\left(\omega_{\sigma}\right)^{m-i} \omega^{i-1}-\sum_{i=2}^{m}(m-i+1)\left(\omega_{\sigma}\right)^{m-i} \omega^{i-1}\right) \operatorname{Ric}(\omega)
$$

and hence the whole right hand side reduces to

$$
\int_{\Gamma_{X}} m \dot{\Phi}_{\sigma} \omega_{\sigma}^{m-1} \operatorname{Ric}(\omega)
$$

Finally, Theorem 5 applies to the variety $\Gamma_{X}$ and gives

$$
\frac{d}{d t} \frac{\mu(Z)}{m+1} \cdot \int_{\Gamma_{X}} \Phi_{\sigma} \sum_{i=0}^{m} \omega^{i} \omega_{\sigma}^{m-i}=\int_{\Gamma_{X}} \dot{\Phi}_{\sigma} \mu(Z) \omega_{\sigma}^{m}
$$


Combining (6.10), (6.11), and (6.12) we obtain

$$
\frac{d}{d t} \nu_{\omega_{Z}}\left(\Phi_{\sigma}\right)=-\frac{1}{V} \cdot \int_{\Gamma_{Z}} \dot{\Phi}_{\sigma}\left(m \operatorname{Ric}\left(\omega_{\sigma}\right)-\mu(Z) \omega_{\sigma}\right) \omega_{\sigma}^{m-1}
$$

This is the derivative of the Mabuchi energy on $Z$. In order to establish Lemma 6.2 we must show it is also the derivative of the Mabuchi energy on $X$ : Replacing $X$ and $Z$ by $\sigma_{0}(X)$ and $\sigma_{0}(Z)$, we see that we may assume that $\sigma_{0}$ is the identity matrix. We shall do that from now on, and we shall write $\dot{\Phi}_{\sigma_{0}}(c)=\dot{\Phi}(c)$ and $\dot{\varphi}_{\sigma_{0}}(c)=\dot{\varphi}(c)$.

Thus, we let

$$
M_{Z}=\operatorname{Ric}\left(\omega_{G r}^{m}\right)-\frac{\mu(Z)}{m} \omega_{G r}
$$

and

$$
M_{X}=\operatorname{Ric}\left(\omega^{n}\right)-\frac{\mu(X)}{n} \omega
$$

We claim that:

$$
\frac{1}{\operatorname{vol}(X)} \int_{X} M_{X} \dot{\varphi}(c) n \omega^{n-1}=\frac{1}{\operatorname{vol}(Z)} \int_{Z} M_{Z} \dot{\Phi}(c) m \omega_{G r}^{m-1}
$$

Theorem 1 then will follow from (6.13) and (6.14).

Let $p_{i}=\left.\pi_{i}\right|_{\Gamma_{X}}$. Then $p_{1}: \Gamma_{X} \rightarrow X$ and $p_{2}: \Gamma_{X} \rightarrow Z$, and we have the following double fibration

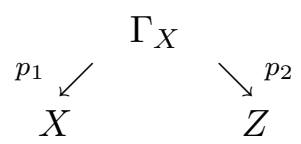

Currents on $X$ and $Z$ can then be compared by pull-backs and push-forths through this fibration. Note that $p_{2}$ is a birational map, that is, there is a Zariski open subset $Z_{0} \subseteq Z$ such that $p_{2}: p_{2}^{-1}\left(Z_{0}\right) \rightarrow Z_{0}$ is bijective. Thus, in terms of pull-backs and push forths

$$
\begin{gathered}
\int_{Z} M_{Z} \dot{\Phi}(c) m \omega_{G r}^{m-1}=\int_{\Gamma_{X}} p_{2}^{*} M_{Z} \cdot p_{2}^{*}\left(\dot{\Phi}(c) m \omega_{G r}^{m-1}\right)= \\
\int_{\Gamma_{X}} p_{1}^{*} M_{X} \cdot p_{2}^{*}\left(\dot{\Phi}(c) m \omega_{G r}^{m-1}\right)+\int_{\Gamma_{X}}\left(p_{2}^{*} M_{Z}-p_{1}^{*} M_{X}\right) \cdot p_{2}^{*}\left(\dot{\Phi}(c) m \omega_{G r}^{m-1}\right)
\end{gathered}
$$

But according to (4.6),

$$
p_{1 *} p_{2}^{*}\left(m \dot{\Phi}(c) \sigma^{*} \omega_{G r}^{m-1}\right)=n \dot{\varphi}(c) \omega^{n-1}
$$


and so we get

$$
\int_{\Gamma_{X}} p_{1}^{*} M_{X} \cdot p_{2}^{*}\left(\dot{\Phi}(c) m \omega_{G r}^{m-1}\right)=\int_{X} M_{X} \dot{\varphi}(c) n \omega^{n-1}
$$

Thus (6.14) will follow from:

$$
\int_{\Gamma_{X}} M(X, Z) \cdot p_{2}^{*}\left(\dot{\Phi}(c) m \omega_{G r}^{m-1}\right)=\int_{X} p_{1 *}\left[M(X, Z) \cdot p_{2}^{*}\left(\dot{\Phi}(c) m \omega_{G r}^{m-1}\right)\right]=0
$$

where

$$
M(X, Z)=\left(p_{2}^{*} M_{Z}-p_{1}^{*} M_{X}\right)
$$

Now $M(X, Z)$ can be made explicit as follows: Define a function $A_{X}: \Gamma_{X} \rightarrow$ $\mathrm{C}$ by the formula

$$
p_{2}^{*} \omega_{G r}^{m}=A_{X} \cdot p_{2}^{*} \omega_{G r}^{m-n} p_{1}^{*} \omega^{n}
$$

Then $M(X, Z)=R\left(h_{X}\right)-\frac{\mu(Z)}{m} p_{2}^{*} \omega_{G r}+\frac{\mu(X)}{n} p_{1}^{*} \omega$ where $h_{X}$ is the curvature of the metric on the relative canonical bundle $\mathcal{L}_{X}=K_{\Gamma_{X}} \otimes p_{1}^{*}\left(K_{X}\right)^{-1}$ given by the formula

$$
h_{X}=A_{X} \cdot p_{2}^{*} \omega_{G r}^{m-n}
$$

We provide now a proof of the key equation (6.16). The main idea is that, by making use of a first jet extension (see the map $\iota_{X}(x)=(x, \zeta)$ defined below), the double fibration (6.15) can be imbedded in another double fibration, which is independent of $X$ and is more symmetric. More precisely, we shall define manifolds $\Sigma$ and $\Gamma^{\prime}$, a fibration $P^{\prime}: \Sigma \rightarrow \Gamma^{\prime}$, a metric $h$ on the relative canonical bundle $\mathcal{L}=K_{\Sigma} \otimes\left(P^{\prime}\right)^{*}\left(K_{\Gamma^{\prime}}\right)^{-1}$ and embeddings $\iota: \Gamma_{X} \hookrightarrow \Sigma$, $\iota_{X}: X \hookrightarrow \Gamma^{\prime}$ which will have the following properties: The projection $p_{1}$ : $\Gamma_{X} \rightarrow X$ is the restriction of $P_{1}$, the metric $h_{X}$ is the restriction of the metric $h$ to $\mathcal{L}_{X}$,

$$
\begin{array}{ccc}
\Gamma_{X} & \rightarrow & \Sigma \\
\downarrow & & \downarrow \\
X & \rightarrow & \Gamma^{\prime}
\end{array}
$$

The reason for doing this is that $\Sigma$ and $\Gamma^{\prime}$ will have a lot of symmetries, which will help us compute the fiber integral of $M$.

Here are the definitions:

$$
\begin{aligned}
& G r=G r\left(N-n-1, \mathbf{P}^{N}\right) \\
& G r^{\prime}=G r\left(n, \mathbf{P}^{N}\right) \\
& \Sigma=\left\{(x, \zeta, z): x \in \mathbf{P}^{N}, \zeta \in G r^{\prime}, z \in G r, x \in \zeta \cap z\right\} \\
& \Gamma=\left\{(x, z): x \in \mathbf{P}^{N}, z \in G r, x \in z\right\}
\end{aligned}
$$


$\Gamma^{\prime}=\left\{(x, \zeta): x \in \mathbf{P}^{N}, \zeta \in G r^{\prime}, x \in \zeta\right\}$

$P^{\prime}(x, \zeta, z)=(x, \zeta)$

$P(x, \zeta, z)=(x, z)$

$\Gamma_{X}=\{(x, z): x \in X, z \in G r, x \in z\}$

$\iota_{X}(x)=(x, \zeta)$ where $\zeta$ is the unique hyperplane of dimension $n$ tangent to $X$ at $x$.

Note that $G=S L(N+1)$ and $U=U(N+1)$ act on $\Sigma, \Gamma, \Gamma^{\prime}, G r, G r^{\prime}$, that $U$ leaves the metrics $\omega, \omega_{G r}, \omega_{G r}^{\prime}$ invariant, and that the original double fibration (6.15) has now been extended to the following double fibration

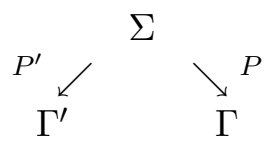

Define a function $A: \Sigma \rightarrow \mathbf{C}$ as follows:

$$
A(x, \zeta, z)=\frac{\omega_{G r}^{m}\left(X_{1}, \ldots, X_{n}, Y_{1}, \ldots, Y_{m-n}\right)}{\omega^{n}\left(X_{1}, \ldots, X_{n}\right) \omega_{G r}^{m-n}\left(Y_{1}, \ldots, Y_{m-n}\right)}
$$

where $a=(x, \zeta, z), Y_{1}, \ldots, Y_{m-n}$ is a basis for the tangent space to the fiber of $P^{\prime}$ above $P^{\prime}(a)$, and $X_{1}, \ldots, X_{n}$ are tangent vectors in $T_{a}(\Sigma)$ whose projections to $\mathbf{P}^{N}$ form a basis of $T_{x}(\zeta)$. This function is clearly invariant under the action of $U$.

Define a metric $h$ on $\mathcal{L}$ by the formula:

$$
h=A \cdot P_{2}^{*} \omega_{G r}^{m-n}
$$

where $P_{2}: \Sigma(x, \zeta, z) \rightarrow z \in G r$ is the projection map. Let $P_{1}: \Sigma(x, \zeta, z) \rightarrow$ $x \in \mathbf{C P}^{N}$, and set

$$
M=R(h)-\frac{\mu(Z)}{m} P_{2}^{*} \omega_{G r}+\frac{\mu(X)}{n} P_{1}^{*} \omega
$$

Then $A_{X}$ and $M(X, Z)$ are respectively the restrictions of $A$ and $M$, and (6.16) is equivalent to

$$
\int_{X} \iota_{X}^{*} P_{1 *}\left[M \cdot P_{2}^{*}\left(\dot{\Phi}(c) m \omega_{G r}^{m-1}\right)\right]=0
$$

In fact, we shall prove that

$$
\iota_{X}^{*} P_{1 *}\left[M \cdot P_{2}^{*}\left(\dot{\Phi}(c) m \omega_{G r}^{m-1}\right)\right]=0
$$


Let $T\left(\Gamma^{\prime}\right)$ be the tangent bundle of $\Gamma^{\prime}$ and let $S \subseteq T\left(\Gamma^{\prime}\right)$ be the subbundle defined by

$$
S=\operatorname{Ker}\left(T\left(\Gamma^{\prime}\right)(x, \zeta) \rightarrow \zeta \in T\left(G r^{\prime}\right)\right)
$$

Thus $S$ is a smooth vector bundle on $\Gamma^{\prime}$ of rank $n$ and for $(x, \zeta) \in \Gamma^{\prime}$, the fiber $S_{(x, \zeta)}$ is the tangent space of $\zeta$ at the point $x$. Since $\iota_{X}\left(T_{x}(X)\right)=S_{(x, \zeta)}$, the relation (6.20) then follows from the following:

$$
\left.P_{1 *}\left[M \cdot P_{2}^{*}\left(\dot{\Phi}(c) m \omega_{G r}^{m-1}\right)\right]\right|_{S}=0
$$

Thus we must show, for every $(x, \zeta) \in \Gamma^{\prime}$, the $(n, n)$ form $P_{1 *}\left[M \cdot P_{2}^{*}\left(\dot{\Phi}(c) m \omega_{G r}^{m-1}\right)\right]$ evaluated at a generator of $\Lambda^{n} T_{x}(\zeta) \otimes \Lambda^{n} T_{x}^{\prime}(\zeta)$, is zero (here $T$ is the holomorphic tangent space and $T^{\prime}$ the anti-holomorphic tangent space).

Thus we fix $(x, \zeta) \in \Gamma^{\prime}$. Recall that $x \in \mathbf{P}^{N}$, that is, $x \subseteq \mathbf{C}^{N+1}$. Also, we have $x \in \zeta$ where $\zeta$ is a plane in $\mathbf{P}^{N}$ of dimension $n$, that is $\zeta \subseteq \mathbf{C}^{N+1}$ is a vector space of dimension $n+1$. The tangent space of $\zeta$ at the point $x$ is canonically isomorphic to $x^{\perp} \cap \zeta=\zeta^{\prime} \subseteq \mathbf{C}^{N+1}$. Let $\zeta_{1}, \ldots, \zeta_{n}$ be an orthonormal basis of $\zeta^{\prime}$. Let

$$
B\left(c, x ; \zeta_{1}, \ldots, \zeta_{n}\right)=P_{1 *}\left[M \cdot P_{2}^{*}\left(\dot{\Phi}(c) m \omega_{G r}^{m-1}\right)\right] \mid\left(\zeta_{1} \wedge \bar{\zeta}_{1}, \ldots, \zeta_{n} \wedge \bar{\zeta}_{n}\right)
$$

We want to show that $B\left(c, x ; \zeta_{1}, \ldots, \zeta_{n}\right)=0$.

Recall that

$$
\dot{\Phi}(c)=\operatorname{tr}\left(\left(c+c^{*}\right) Z Z^{*}\right)
$$

where, abusing notation, $Z$ is an $(N+1) \times(N-n)$ matrix whose columns form an orthonormal basis of the vector space $Z \subseteq \mathbf{C}^{N+1}$.

Define an $(N+1) \times(N+1)$ matrix

$$
B(x, \zeta)=B\left(x ; \zeta_{1}, \ldots, \zeta_{n}\right)=P_{1 *}\left[M \cdot P_{2}^{*}\left(Z Z^{*} m \omega_{G r}^{m-1}\right)\right] \mid\left(\zeta_{1} \wedge \bar{\zeta}_{1}, \ldots, \zeta_{n} \wedge \bar{\zeta}_{n}\right)
$$

Then $B\left(c, x ; \zeta_{1}, \ldots, \zeta_{n}\right)=\operatorname{tr}\left(\left(c+c^{*}\right) B(x, \zeta)\right)$ so it suffices to show that $B(x, \zeta)=0$ for all $(x, \zeta) \in \Gamma^{\prime}$.

Note that $B$ has the following properties:

$$
B(u(x, \zeta))=u B(x, \zeta) u^{*}, \quad B\left(x, \zeta u_{1}\right)=B(x, \zeta)
$$

for all $u \in U(N+1)$ and all $u_{1} \in U(n)$. This implies that

$$
B(x, \zeta)=k \cdot \zeta \zeta^{*}
$$


for some constant $k$. We claim that $k=0$. To see this, we take the trace of (6.22) and use the fact that $\operatorname{tr}\left(\zeta \zeta^{*}\right)=\operatorname{tr}\left(\zeta^{*} \zeta\right)=(n+1)$ since $\zeta \zeta^{*}$ is the identity matrix, and that likewise, $\operatorname{tr}\left(Z Z^{*}\right)=\operatorname{tr}\left(Z^{*} Z\right)=(N-n)$ :

$$
\begin{gathered}
k \cdot(n+1) \pi^{*} \omega_{F S}^{n}\left(\zeta_{1} \wedge \bar{\zeta}_{1}, \ldots, \zeta_{n} \wedge \bar{\zeta}_{n}\right) \\
=P_{1 *}\left[M \cdot P_{2}^{*}\left((N-n) m \omega_{G r}^{m-1}\right)\right] \mid\left(\zeta_{1} \wedge \bar{\zeta}_{1}, \ldots, \zeta_{n} \wedge \bar{\zeta}_{n}\right)
\end{gathered}
$$

where $\pi: \Gamma^{\prime} \rightarrow \mathbf{P}^{N}$ is the projection map. Applying $\iota_{X}^{*}$ to both sides and integrating over $X$ we get

$$
k \cdot(n+1) \cdot \int_{X} \omega_{F S}^{n}=(N-n) \int_{\Gamma_{X}}\left(p_{2}^{*} M_{Z}-p_{1}^{*} M_{X}\right) p_{2}^{*} \omega_{G r}^{m-1}
$$

But

$$
\int_{\Gamma_{X}} p_{2}^{*} M_{Z} \cdot p_{2}^{*} \omega_{G r}^{m-1}=\int_{Z} M_{Z} \cdot \omega_{G r}^{m-1}=0
$$

Also, making use of (4.7) we have

$$
\int_{\Gamma_{X}} p_{1}^{*} M_{X} \cdot p_{2}^{*} \omega_{G r}^{m-1}=\int_{X} M_{X} \cdot p_{1 *} p_{2}^{*} \omega_{G r}^{m-1}=D_{1} n \int_{X} M_{X} \cdot \omega_{F S}^{n-1}=0
$$

Thus $k \cdot(n+1)=0-0$ so $k=0$. Thus $B(x, \zeta)=0$ and the proof of Lemma 6.2, and hence of Theorem 1 is complete. Q.E.D.

Remark. Although we do not require it in the preceding proof, it may be useful to note that the key function $A(x, \zeta, z)$ introduced can be described by a simple explicit formula. Let $x^{\perp} \subseteq \mathbf{C}^{N+1}$ be the orthogonal complement of $x$, and let $\pi_{x}: \mathbf{C}^{N+1} \rightarrow x^{\perp}$ be the orthogonal projection. Then $\pi_{x}(\zeta) \subseteq x^{\perp}$ has dimension $n$ and $\pi_{x}(z) \subseteq x^{\perp}$ has dimension $N-n-1$. These two spaces will generically span a subspace $\pi_{x}(\zeta+z)$ of codimension 1 inside $x^{\perp}$. Then $A(x, \zeta, z)$ is the length of the Plücker vector of $\pi_{x}(\zeta+z)$ with respect to a basis which is the union of an orthonormal basis for $\pi_{x}(z)$ and $\pi_{x}(\zeta)$

$$
A(x, \zeta, z)=\frac{\left|P l\left(\pi_{x}(\zeta+z)\right)\right|}{\left|P l\left(\pi_{x}(\zeta)\right)\right| \cdot\left|P l\left(\pi_{x}(z)\right)\right|}
$$

Another way of writing this is as follows. Fix $x$ and let $H=x^{\perp}$. View $A$ as a function on $\operatorname{Gr}(n, H) \times G r(N-n-1, H)$. Let

$$
\theta: G r(n, H) \times G r(N-n-1, H) \rightarrow G r(N-1, H)
$$


be the map which sends $\left(\zeta_{0}, z_{0}\right)$ to the space spanned by $\zeta_{0}$ and $z_{0}$. Then

$$
-\frac{\sqrt{-1}}{2 \pi} \partial \bar{\partial} \log A=\theta^{*} \omega_{N-1}-\omega_{n}-\omega_{N-n-1}
$$

where the $\omega_{k}$ are just Fubini-Study metrics. In other words, $R(h)=$ $\theta^{*} \omega_{N-1}-\omega_{n}$.

Remark. Generalized Mabuchi energies for singular varieties emerge naturally from the above proof, and may be worth investigating in their own right. Related extensions for the Futaki invariant were studied in Ding and Tian [DT].

Remark. There are many ways of expressing the current $\left[Y_{s}\right]$. It is intriguing that the term it produces can be formally viewed as an $F^{0}$ functional for $Y_{s}$.

Remark. The genericity assumption on $X$ is not really restrictive. It should suffice for the study of asymptotic stability, when the variety $X$ is imbedded into projective space by the bases of the antipluricanonical bundle $K_{X}^{-p}$ for $p$ large.

Remark. The seminorm $\|\cdot\|_{\#}$ is degenerate. Its main property is that for any norm $\|\cdot\|_{B}$ on $H^{0}(G r, O(d))$ with $\|f\|>0$ for $f \neq 0$, there exists a constant $C>0$ so that

$$
\|f\|_{\#} \leq C\|f\|_{B}
$$

for all $f \in H^{0}(G r, O(d))$. This follows from the continuity of $\|\cdot\|_{\#}$, which requires a somewhat technical argument. For our purposes, it suffices to observe that the second term in the expression (1.1) for $\|\cdot\|_{\#}$ is continuous, since the only possible divergences of the integrand is logarithmic. As for the first term, we can estimate it as follows

$$
\int_{Z} \log \left(\omega_{G r}^{m} \wedge \partial \bar{\partial}\left(\frac{|f(z)|^{2}}{|P \ell(z)|^{2 d}}\right)\right) \wedge \omega_{G r}^{m} \leq C \sup _{G r} \log |\nabla f|^{2} \int_{Z} \omega_{G r}^{m}
$$

Since the logarithmic is an increasing function and $\sup _{G r} \log |\nabla f|^{2}=$ $\log \sup _{G r}|\nabla f|^{2}$, the right hand side is bounded on the unit ball with respect to $\|\cdot\|_{B}$ in $H^{0}(G r, O(d))$, and our claim follows.

Remark. As mentioned in $\S 5$, the Deligne Pairing is related to the concept of CM stability, as defined by Tian [T2]:

Let $G=S L(N+1, \mathbf{C})$. Let $\pi: \mathcal{X} \rightarrow B$ be a $G$ equivariant holomorphic fibration between smooth varieties, equivariantly embedded in $B \times \mathbf{P}^{N}$. Tian 
constructs a $G$ equivariant line bundle $L_{B}$ over $B$ and a metric $\|\cdot\|_{Q}$ on $L_{B}$ with the following property: Let $b \in B$ and let $X=\pi^{-1}(b)$.

$$
\nu_{\omega}\left(\varphi_{\sigma}\right)-\psi(\sigma)=C \cdot \log \frac{\|\sigma \cdot b\|_{Q}^{2}}{\|b\|_{Q}^{2}}
$$

for some positive constant $C$, where $\psi$ is a certain explicitly defined function which is bounded above.

Now we let $\mathcal{K}$ be the relative canonical bundle of $\mathcal{X}$ over $B$ and let $\mathcal{L}$ be the $O(1)$ bundle on $\mathcal{X}$. For $r$ and $s$ integers, let

$$
\mathcal{M}=\left\langle\mathcal{K}, \mathcal{L}, \mathcal{L}, \ldots, \mathcal{L}>^{r}(\mathcal{X} / B) \otimes<\mathcal{L}, \mathcal{L}, \mathcal{L}, \ldots, \mathcal{L}>^{s}(\mathcal{X} / B)\right.
$$

where the first factor is the Deligne pairing of the bundle $\mathcal{K}$ and $n$ copies of the bundle $\mathcal{L}$ and the second is the Deligne pairing of $n+1$ copies of the bundle $\mathcal{L}$. Then $\mathcal{M}$ is a $G$ equivariant line bundle over $B$ and comes equipped with the Deligne metric $\|\cdot\|_{D}$. Let $b \in B$ and let $X=\pi^{-1}(b)$. Then, for suitably chosen $r$ and $s$, we can show that

$$
\nu_{\omega}\left(\varphi_{\sigma}\right)=C \cdot \log \frac{\|\sigma \cdot b\|_{D}^{2}}{\|b\|_{D}^{2}}
$$

It is natural to compare (6.27) and (6.28) and investigate their relationship. Also, the role of the pairing $<\mathcal{K}, \mathcal{K}, \ldots, \mathcal{K}, \mathcal{L}, \mathcal{L}, \ldots, \mathcal{L}>(\mathcal{X} / B)(p$ copies of $\mathcal{K}$ and $q$ copies of $\mathcal{L}$ with $p+q=n+1$ ) needs to be clarified. These questions will be addressed in an upcoming paper.

\section{Acknowledgements.}

The authors would like to thank Gang Tian for many very helpful suggestions, especially on singularities, and Sean Paul for calling their attention to important examples of unstable manifolds. They would also like to thank J. Song, Mu-Tao Wang, Ben Weinkove, and all the members of the Columbia geometric analysis group for many stimulating exchanges.

\section{References.}

[BM] Bando, S. and T. Mabuchi, "Uniqueness of Einstein-Kähler metrics modulo connected group actions", in Algebraic Geometry, Sendai 1985, Adv. Stud. in Pure Math. 10 (1987) 11-40, Kinokuniya, Tokyo and North-Holland, Amsterdam. 
[C1] Chen, X., "On the lower bound of the Mabuchi energy and its application", Int. Math. Res. Notices 12 (2000) 607-623.

[C2] Chen, X., "The space of Kähler metrics", J. Differential Geom. 56 (2000) 189-234.

[De] Deligne, P., "Le determinant de la cohomologie", Contemporary Math. 67 (1987) 93-177.

[DT] Ding, W. and G. Tian, "Kähler-Einstein metrics and the generalized Futaki invariants", Inventiones Math. 110 (1992) 315-335.

[D1] Donaldson, S., "Anti self-dual Yang-Mills connections over complex algebraic surfaces and stable vector bundles", Proc. London Math. Soc. 50 (1985) 1-26.

[D2] Donaldson, S., "Scalar curvature and projective imbeddings I", 2001 Preprint.

[D3] Donaldson, S., "Infinite determinants, stable bundles, and curvature", Duke Math. J. 54 (1987) 231-247.

[F] Futaki, A., "On a character of the automorphism group of a compact complex manifold", Inventiones Math. 87 (1987) 655-660.

[KN] Kempf, G. and L. Ness, "The length of vectors in representation spaces", Algebraic Geometry (Proc. 1978 Summer Meeting, Copenhagen), Lecture Notes in Math. 732 (1979) 233-243, Springer-Verlag.

[Lu1] Lu, Z., "On the lower order terms of the asymptotic expansion of Tian-Yau-Zelditch", Amer. J. Math. 122 (2000) 235-273.

[Lu2] Lu, Z., "K-energy and K-stability for hypersurfaces", math.DG/0108009

[Luo] Luo, H., "Geometric criterion for Gieseker-Mumford stability of polarized manifolds", J. Diff. Geom. 49 (1998) 577-599.

[M] Mabuchi, T., "K-energy maps integrating Futaki invariants", Tohoku Math. J. 38 (1986) 245-257.

[Mu] Mumford, D., "Stability of projective varieties", L'Enseignement Mathematique, 23 (1977) 39-110. 
[P] Paul, S. "Geometric analysis of Chow Mumford stability", Princeton Ph.D. Thesis (2000).

[SY] Siu, Y.T. and S.T. Yau, "Complete Kähler manifolds with nonpositive curvature of faster than quadratic decay", Ann. of Math. 105 (1977) $225-264$.

[T1] Tian, G., "The K-energy on hypersurfaces and stability", Comm. Anal. Geometry 2 (1994) 239-265.

[T2] Tian, G., "Kähler-Einstein metrics with positive scalar curvature", Inventiones Math. 130 (1997) 1-37.

[T3] Tian, G., "Canonical Metrics in Kähler Geometry", Birkhäuser, Basel, 2000 .

[T4] Tian, G., "Bott-Chern forms and geometric stability", Discrete Contin. Dynam. Systems 6 (2000) 211-220.

[UY] Uhlenbeck, K. and S.T. Yau, "On the existence of Hermitian YangMills connections on stable vector bundles", Commun. Pure Appl. Math. 39 (1986) 257-293.

[W] Wang, X., "Balance point and stability of vector bundle over projective manifolds", Brandeis 2001 preprint.

[Y1] Yau, S.T., "On the Ricci curvature of a compact Kähler manifold and the complex Monge-Ampere equation I", Comm. Pure Appl. Math. 31 (1978) 339-411.

[Y2] Yau, S.T., "Open Problems in Geometry", Proc. Symposia Pure Math. 54 (1993) 1-28.

[Y3] Yau, S.T., "Review of Kähler-Einstein metrics in Algebraic Geometry", Israel Math. Conf. Proceedings 9 (1996) 433-443.

[Y4] Yau, S.T., "Nonlinear analysis in geometry", Enseign. Math. 33 (1987) 109-158.

[Z] Zhang, S., "Heights and reductions of semi-stable varieties", Compositio Math. 104 (1996) 77-105.

D. H. PHONG

Department of Mathematics

Columbia University, New York, NY 10027 
JACOB STURM

Department of Mathematics

Rutgers University, Newark, NJ 07102

Received OCtober 25, 2002. 